\title{
Parking search equilibrium on a network
}

\author{
Stephen D. Boyles * $\quad$ Shoupeng Tang ${ }^{\dagger} \quad$ Avinash Unnikrishnan ${ }^{\ddagger}$
}

August 16, 2014

\begin{abstract}
This paper describes an equilibrium formulation for incorporating parking search into traffic network assignment models. The proposed model allows general network topologies and reflects uncertainty related to parking availability, including the possibility of cycling behavior as drivers search for parking. The equilibrium framework represents the mutual dependence between the probabilities of finding parking at different locations and the search processes employed by drivers to minimize total expected journey time (or cost). In this framework, network loading is represented by a system of nonlinear flow conservation networks, and feasibility and uniqueness issues are discussed. The equilibrium problem is formulated as a variational inequality and a convex combinations heuristic is proposed. Numerical results show that neglecting parking search can substantially underestimate network flows, and quantitatively demonstrate the relationship between parking duration effects and the cost of time spent walking relative to driving, and the expected driving and walking times.
\end{abstract}

Keywords: Network equilibrium; parking search; nonlinear systems

\section{Introduction}

Travel forecasting models are used to predict traffic patterns and overall system-level congestion for evaluating transportation system improvement measures. One of the key steps in travel forecasting models is traffic assignment, which predicts the route choices of travelers and the resulting flows on the networks (Patriksson, 1994). Parking is an underappreciated aspect of transportation network modeling. While some studies conclude that over a third of traffic volume can be attributed to drivers searching for parking (Shoup, 2006), most traffic assignment models used in practice neglect additional time at the destination due to parking search. Integrating the parking search process into network traffic assignment models will result in better prediction of traffic flows, leading to more accurate evaluation or rankings of transportation improvement projects, such as where to add capacity, pricing, and so forth (Meng et al., 2001; Boyce et al., 2004; Gardner et al., 2011). Many

\footnotetext{
*Assistant Professor, Department of Civil, Architectural \& Environmental Engineering, The University of Texas at Austin, 301 E. Dean Keeton St. Stop C1761, Austin, TX 78712, Ph: 512-471-3548, Fax: 512-475-8744, Email: sboyles@mail.utexas .edu

${ }^{\dagger}$ Ph.D. Candidate, Department of Civil, Architectural \& Environmental Engineering, The University of Texas at Austin

${ }^{\ddagger}$ Assistant Professor, Department of Civil and Environmental Engineering, West Virginia University
} 

dynamic pricing based on parking availability (Rye, 2006; Polycarpou et al., 2013; Glasnapp et al., 2014). Recent advances in sensor technologies and parking-related smartphone apps also suggest the value of a quantitative framework for calculating the potential impacts of these technologies as drivers adapt their parking behavior in response.

As with route choice in general, parking search can be viewed from the perspective of equilibrium. Assuming that drivers aim to minimize the time spent traveling (including both driving and walking from the parking space to the destination), drivers' route and parking search behaviors depend on the probabilities of finding parking at particular locations in the network; however, these probabilities depend on the route and search strategies employed by drivers in the network. A natural model for this mutual dependency is an equilibrium framework in which no driver can improve his or her expected travel time by adjusting their strategy.

As discussed in the literature review, the model presented in this paper builds on existing network parking models in the following ways. First, it is explicitly stochastic and reflects the dependence of parking probability on searching rates. Second, it applies to general networks of any topology, and directly allows planners to identify which specific links and regions are particularly affected by increases in volume due to parking search. Third, the concepts of route choice and parking search are unified in a natural way which does not require assumptions such as drivers "transitioning" from driving towards the destination to searching for parking. Fourth, the introduction of an equilibrium concept captures the dependency between searching strategies and parking availability.

To accomplish this, a network transformation is introduced to distinguish between drivers searching for parking on a link and drivers merely passing through. The dependence of parking probability on flow rates results in a set of nonlinear flow conservation equations. Nevertheless, as shown below, under relatively weak assumptions the existence and uniqueness of the network loading can be shown, and an intuitive "flow-pushing" algorithm can be used to solve for the solution of this nonlinear system. Built on this network loading algorithm, travel times can be computed. The equilibrium is formulated as a variational inequality, and a heuristic algorithm is presented to solve it.

The remainder of the paper is organized as follows. Section 2 reviews relevant literature on the impact of parking in urban areas, along with network modeling approaches which have been proposed. Section 3 introduces the network transformation used to represent the stochastic nature of the parking search and notation which will be used throughout. Network loading and flow conservation are described in Section 4, along with the flow-pushing algorithm; this section describes the impact of travel choices on parking availability and link flows. Next, Section 5 introduces the complementary perspective of the impact of parking availability on travel choices, leading to an equilibrium definition to reconcile both perspectives. A solution heuristic is presented in this section as well. Section 6 demonstrates the algorithm's performance numerically and conducts sensitivity analyses, while Section 7 concludes and discusses future directions. 


\section{Literature Review}

Parking imposes significant demands on urban transportation networks. Drivers "cruising" or searching for parking increase roadway volumes, exacerbating congestion and emissions. A metaanalysis by Shoup (2006) finds that approximately 34\% of congestion in urban areas results from cruising for parking, and a study in Frankfurt, Germany indicates that up to $40 \%$ of total travel time consists of searching for a parking space, for peak-period trips to the city center (Axhausen et al., 1994). As a result, many cities are focusing attention on parking management, including data collection, real-time dynamic pricing, and other strategies; such cities include San Francisco (Pierce and Shoup, 2013), Boston (Ross, 2013), Seattle, and Washington, DC (Greenberg, 2012).

Network models that incorporate parking can be broadly classified into simulation-based approaches and analytic approaches. Simulation approaches include the study of Thompson and Richardson (1998), in which drivers choose parking spaces based on a disutility function incorporating time and cost variables, and agent-based approaches (Benenson et al., 2008; Gallo et al., 2011; Dieussart et al., 2009) in which drivers are assigned behavioral rules. While simulation has the advantage of explicitly modeling parking dynamics and accommodating behavioral heterogeneity, they are limited in their ability to model large networks and are generally not amenable to exact results regarding the network loading and the equilibrium state. A further limitation is that in the absence of field data, there is an arbitrary element to the behavior rules, such as assuming that drivers will not cruise for parking if vacant spaces are available (Arnott et al., 1991), or that drivers will route deterministically to a preferred parking location; if that choice is unavailable, they will proceed to a second choice, third choice, and so on (Leurent and Boujnah, 2012). While some degree of arbitrariness is inevitable without field data, we prefer to build a model on a more fundamental principle. As described below, in our model the route choice and choice whether to take an available space if one exists are both governed by the principle of expected cost minimization, without the need to introduce a distinction between "driving toward the destination" and "searching for a parking space."

Analytical approaches, by contrast, are based on traffic assignment concepts and transform the network by adding new links to represent parking options. Typically these links are equipped with an impedance function to reflect delay due to parking search as more drivers attempt to park on that link. These approaches include Eldin et al. (1981), Lam et al. (2002), Lam et al. (2006), and Li et al. (2007), and incorporate features such as endogenous mode choice accounting for parking, bilevel models for parking price. The main advantage of these approaches is their tractability, and ability to incorporate well-known results from the traffic assignment literature. However, by assuming a deterministic impedance for parking, the models are unable to reflect additional delay or volume on specific network links as drivers search for parking (possibly traversing a link multiple times as they cycle). Discrete choice concepts have also been used to study parking choice (Hunt and Teply, 1993), without explicit reference to a network, but using a nested logit model to account for similarities in on-street and off-street alternatives.

By contrast, the model described in this paper is explicitly stochastic and can be used to identify specific links with increased volume due to parking search. Furthermore, the network loading can be described analytically, and the equilibrium principle can be formulated mathematically. Furthermore, the behavior model relies on a fairly simple principle (travel time minimization) and does not require exogenous assumptions on when drivers begin searching for parking (which are common in agent-based simulation models). In this way it is similar to the model of Arnott and 
Rowse (1999), but allows for general network topologies, as opposed to assuming the network is a homogeneous circle, and does not require introducing a "cruising distance" from the destination, where drivers accept any vacant space within this threshold and reject any vacant space outside it. However, in contrast to Arnott and Rowse (1999) the current paper does not attempt to quantify the value of parking information systems or other parking-related policies, which seems challenging in more general network topologies.

Similarly, other researchers have studied parking-related policies, such as optimal pricing and control of parking spaces (Qian and Rajagopal, 2014), more general pricing problems with a single bottleneck for congestion (Zhang et al., 2008; Qian et al., 2012; Yang et al., 2013), and distribution of permits for parking (Zhang et al., 2011). In order to focus on these policies, these researchers studied the parking problem in relatively stylized settings, such as a single bottleneck, a linear homogeneous city, or with two parallel alternatives. This paper presents a complementary approach by focusing on the issues associated with a generic network topology, and aims to provide a foundation for extending the results of these earlier papers to more general networks.

An analogy can be drawn between the parking model developed in this paper and the user equilibrium with recourse model (Unnikrishnan and Waller, 2009), in which travelers are routed stochastically based on realized network states. Indeed, both models have as a subproblem the online shortest path problem (Waller and Ziliaskopoulos, 2002; Provan, 2003), as recognized in Tang et al. (2014). The primary distinction is that the routing probabilities in user equilibrium with recourse are flow-independent, whereas in the parking model routing probabilities depend on flow to reflect the dependence of parking availability on searching intensity. This results in nonlinear flow conservation equations, which require more finesse. There is also a passing similarity to the equilibrium model developed in Nie (2011), in which the probability distributions of link travel times are flowdependent; the primary distinction is that in the current paper the flow-dependent stochasticity affects the routing of vehicles, not just the time experienced. The following sections describe the model and modifications in more detail.

\section{$3 \quad$ Network structure}

Consider a transportation network $G=(\mathcal{N}, \mathcal{A})$ with node and $\operatorname{arc} \operatorname{sets} \mathcal{N}$ and $\mathcal{A}$, respectively. The parking process is modeled using a network transformation (Figure 1) to represent potential parking availability. To represent this transformation, the node and arc sets are partitioned: the nodes $\mathcal{N}$ are divided into disjoint subsets $N_{R}, N_{P}, N_{D}$, and $N_{T}: N_{R}$ represents the "regular" intersection nodes (the traditional nodes in most transportation network models), $N_{P}$ represents the parking nodes (one for each link), $N_{D}$ the destination nodes, and $N_{T}$ the transition nodes connecting parked flows to the respective destinations. Notice that the destination nodes are connected to the intersection nodes only through parking and transfer nodes. We aim to model a scenario where vehicles are traveling towards a region where parking is scarce (e.g., home to downtown), and assume that the return trip (downtown to home) does not involve a search for on-street parking.

Similarly, the arcs $\mathcal{A}$ are divided into disjoint subsets $A_{R}, A_{S}, A_{P}, A_{N P}$, and $A_{T}$. These $\operatorname{arcs}$ can represent both on-street parking and off-street lots or garages. $A_{R}$ represents "regular" or thru links, the traditional arcs in network models (the physical roadway infrastructure), and flow on this arc represents drivers who are not looking for parking. Each $\operatorname{arc}(i, j)_{R} \in A_{R}$ is associated with one 


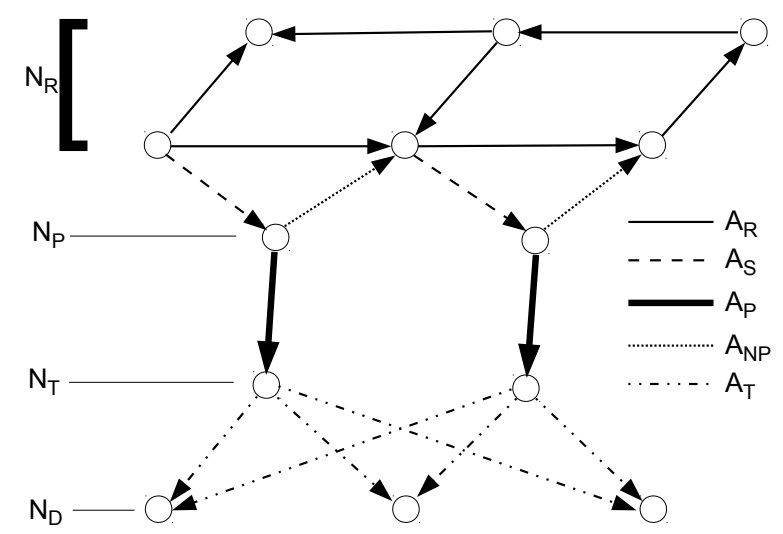

Figure 1: Linkages and layers between nodes and arcs for modeling parking.

arc in each of the remaining subsets: flow on the corresponding arc $(i, j)_{S} \in A_{S}$ represents drivers who are searching for parking on link $(i, j)$ and will park there if a space is available. The arc $(i, j)_{P} \in A_{P}$ is used to represent drivers who are actually able to find a space, and $(i, j)_{N P} \in A_{N P}$ drivers who are unable to find a space. Finally the links $(i, j)_{T}^{d}$ connect flow parked on link $(i, j)$ to destination $d$. As a notational convention, we use $(i, j) \in \mathcal{A}$ and the subscript $i j$ in equations whenever the type of arc (regular, parking, etc.) is irrelevant or when multiple types of arcs are meant. When only a single type of arc is intended, the links $(i, j)_{R},(i, j)_{S},(i, j)_{P}$, and $(i, j)_{T}^{d}$ are referred to with the subscripts $i j, R ; i j, S ; i j, P$; and $i j, T, d$, respectively.

Notice that each node in $N$ may have incoming links from $A_{R}$ and $A_{N P}$ and outgoing links from $A_{R}$ and $A_{S}$; each node in $N_{P}$ has a single incoming link from $A_{S}$ and two outgoing links, one from $A_{P}$ and one from $A_{N P}$; each node in $N_{D}$ has incoming links from $A_{T}$ and no outgoing links; and each node in $N_{T}$ has a single incoming link from $A_{P}$ and outgoing links from $A_{T}$. We further assume that an arc exists between each transfer node and every destination. The interpretation is that it is always possible to walk from any link to the destination, although perhaps with high disutility.

Each link is associated with the steady-state flow rate of vehicles on this link: this value is denoted $x_{i j}$. Each node is associated with a flow conservation constraint relating the flow values on the incoming and outgoing links. These flow conservation constraints for our model differ from standard flow conservation constraints in two ways: (1) flow is split at parking nodes $N_{P}$ based on the (flowdependent) availability of parking spaces; and (2) flow is proportionately split at intersection nodes $N$ to reflect the parking search process. Flow conservation equations have slightly different form for different types of nodes. Flow conservation for nodes in $N_{P}$ is described in Section 4.1 along with the model for parking availability probabilities. Flow conservation for nodes in $N_{R}$ is described in Section 4.2 along with the description of user behavior. Flow conservation for $N_{T}$ and $N_{D}$ nodes are straightforward and found in that subsection as well.

Each link is also equipped with a generalized cost $t_{i j}$ intended to reflect the total disutility of travel on that link (including time, cost, and other factors). For instance, additional disutility may be added to searching links to represent slower travel or more mental exertion and stress compared to thru driving. To simplify the notation these values are assumed constant and independent of flow. This is not restrictive and all of the algorithms and results in this paper immediately transfer to the case of flow-dependent costs, even when the mapping from flow to cost is not separable (since 

continuous.

The use of the word "steady-state" to describe the flows (and later, parking probabilities) merits discussion. It is assumed that the rates at which vehicles enter links (both searching for parking and driving through), and the rate at which parked vehicles vacate spaces to return home, are constant on each link, although of course these rates may differ for different links. Under the assumptions made on the type of trips being modeled and the network - particularly, that we are modeling trips with a single stop in a region with limited parking, that the return journey home does not involve stochastic parking search, and that link costs are flow-independent — vehicles on "return" trips can be ignored for purposes of estimating parking probabilities and increased volume from parking search, which is the focus of this paper.

Our behavior model assumes that drivers make all parking-related choices to minimize the expected total cost of travel. In this paper we assume that drivers can be aggregated by destination, which is consistent with this assumption. By linearity, any travel which has occurred between the origin and a driver's current location is a "sunk cost" which should not affect the decisions between this current location and the destination; therefore at any location, under the steady-state assumption, all drivers with the same destination face the same set of choices with the same set of costs.

Furthermore, the only place in our transformed network where drivers exercise "choice" is at the intersection nodes $N_{R}$. At parking nodes, the split of drivers is determined entirely by the parking probabilities, and at transfer nodes, all drivers head to the appropriate destination, as specified below. We model behavior through the use of splitting proportions, in which the fraction of drivers arriving at a node and heading to a common destination, and departing on a link (either a parking search or thru link), is specified.

These have a similar interpretation to the flow proportions in Bar-Gera (2002) and Gentile (2009), but differ because those works identify acyclic bushes in which these proportions identify path flows directly. The parking search process described here may involve cycles, because parking availability on any link is stochastic and cannot be predicted in advance. This issue is discussed in more detail in the following sections. As a preview, Section 4 introduces the notion of strong feasibility, which ensures that a unique solution exists to the nonlinear flow conservation equations. Section 5 then introduces a new feasibility notion which proves more practical for formulating and solving the equilibrium version of the problem, although the behavioral interpretation is slightly less elegant.

\section{Network loading}

This section has three major goals: (1) formally provide the set of flow conservation equations (including representations of the probability of successfully finding parking on a link and user choice at nodes); (2) define the notion of strong feasibility and show existence and uniqueness of solutions to the flow conservation equations, which is nontrivial since they are nonlinear; and (3) provide a simple "flow-pushing" algorithm for solving this nonlinear system and demonstrate its correctness under nonrestrictive assumptions. 


\subsection{Parking availability probability}

Associated with each searching link $(i, j)_{S} \in A_{S}$ is a function $p_{i j}: \mathbb{R}^{+} \rightarrow[0,1]$ representing the probability that a vehicle seeking to park on this link will find an available space. Particularly, $p_{i j}\left(x_{i j}^{S}\right)$ reflects the proportion of vehicles seeking to park on $(i, j)$ that are able to, and $x_{i j}^{S} p_{i j}\left(x_{i j}^{S}\right)$ reflects the rate at which vehicles are parking on this link. For convenience, the functions $f_{i j}(x)=$ $x p_{i j}(x)$ are defined to express this latter rate. (If parking is not allowed on this link $p_{i j}(x) \equiv 0$.) This is expressed through the flow conservation constraints which apply to each parking node:

$$
\begin{aligned}
x_{i j}^{P} & =f_{i j}\left(x_{i j}^{S}\right) & \forall(i, j)_{P} \in A_{P} \\
x_{i j}^{N P} & =x_{i j}^{S}-x_{i j}^{P} & \forall(i, j)_{N P} \in A_{N P}
\end{aligned}
$$

The formulation is general and given in terms of an arbitrary function $p_{i j}$. However, it is reasonable to assume that each function $p_{i j}$ is nonincreasing (the more vehicles searching for parking, the lower the chance each will find an available space), but that $f_{i j}$ is strictly increasing (the more vehicles searching for parking, the higher the total rate of parking vehicles will be even as the probability any specific vehicle can find a space decreases). Furthermore, these assumptions are used to establish uniqueness of the solution to the flow conservation equations and convergence of the network loading algorithm. If $p_{i j}(x)$ is differentiable, then $0<\frac{d}{d x} f_{i j}(x) \leq 1$ is sufficient for these assumptions to be satisfied. While we believe this assumption to be reasonable, the specific results given below will use weaker conditions when possible.

For each parking link $(i, j)_{P}$, define its parking capacity to be

$$
C_{i j, P}=\sup _{x}\left\{f_{i j}(x)\right\}
$$

reflecting an upper bound on the rate at which vehicles can leave this link.

In practice, this function is likely related to the number of available parking spaces on a link and the duration vehicles remain parked there. For instance, if a link has $S$ parking spaces, and the parking duration is distributed exponentially with mean $\mu$, then the probability a given vehicle will find an available space when vehicles are searching for parking on that link at rate $x$ is

$$
p(x)=\frac{\sum_{k=0}^{S-1}(\mu x)^{k} / k !}{\sum_{k=0}^{S}(\mu x)^{k} / k !}
$$

as derived in the Appendix, which also includes an approximation to facilitate computation when $S$ is large.

\subsection{Routing and parking search strategies}

As introduced above, both route choice and the choice of parking in a vacant space (if available) are described with splitting fractions at each node. In particular, let $\alpha_{i j}^{d}$ reflect the fraction of travelers arriving at node $i$ en route to destination $d$ who choose to exit on link $(i, j)$. If this link is a regular link, the driver will not park on this link even if an available space is found (presumably in hopes of parking on a link with a shorter walking distance), and this driver will not affect the probability 
of a parking space being available. On the other hand, if $(i, j)$ is a searching link, then the driver will park on this link should a space become available.

Define the set of weakly feasible splitting proportions

$$
\Omega=\left(\boldsymbol{\alpha} \in \mathbb{R}_{+}^{\left|N_{D}\right|\left(\left|A_{R}\right|+\left|A_{S}\right|\right)}: \sum_{(i, j) \in \mathcal{A}} \alpha_{i j}^{d}=1 \quad \forall i \in N_{R}, d \in N_{D}\right)
$$

As the name suggests, there are additional feasibility concerns beyond these obvious requirements on $\boldsymbol{\alpha}$, discussed in the next section.

Let $q_{i d}$ denote the demand for travel to destination $d \in N_{D}$ from node $i \in N_{R}$, expressed as a rate in the same units as $x$. Let the superscript $d$ on a flow variable index the destination-disaggregated flow; for instance $x_{i j}^{d}$ is the flow on link $(i, j)$ destined for node $d \in N_{D}$. Clearly

$$
x_{i j}=\sum_{d \in N_{D}} x_{i j}^{d}
$$

for all $(i, j) \in \mathcal{A}$. The flow proportions are given by $\alpha_{i j}^{d}$, representing the fraction of drivers arriving at node $i$ and heading for destination $d$ which will choose link $(i, j) \in \mathcal{A}$. This gives the disaggregate flow conservation equations for intersection nodes:

$$
x_{i j}^{d}=\alpha_{i j}^{d}\left(q_{i d}+\sum_{(h, i) \in \mathcal{A}} x_{h i}^{d}\right) \quad \forall i \in N_{R},(i, j) \in \mathcal{A}, d \in N_{D}
$$

Flow conservation equations for transfer nodes and destination nodes follow trivially:

$$
\begin{aligned}
x_{i j, T, d}^{d} & =x_{i j, P}^{d} \\
x_{i j, T, e}^{d} & =0 \\
\sum_{(i, j)_{T} \in A_{T}} x_{i j, T, d}^{d} & =\sum_{i \in N_{R}} q_{i d} \\
\forall(i, j) \in A_{T} ; d, e \in N_{D} ; d \neq e & \forall d \in N_{D}
\end{aligned}
$$

In what follows, references to "the flow conservation equations" are to the collection of equations (1), (2), (7), (8), (9), and (10).

As an example of these concepts, refer to panels (a) and (b) of Figure 2. Panel (a) shows a network with two parking options from the origin $i$ to destination $d$ : parking along link $(i, j)$ is closer to the origin, but vehicles can only park successfully with probability of $1 / 2$ (for illustrative purposes, assumed constant and independent of searching intensity). Link $(k, m)$ is further away, but parking is assured. Panel (b) shows the network loading corresponding to $\alpha_{i j, S}=2 / 3, \alpha_{i k}=1 / 3, \alpha_{j i}=$ $\alpha_{k m, S}=1$, and all other components of $\boldsymbol{\alpha}$ zero. The reader may verify that the flow conservation equations are satisfied for each link in this network, and note that half of the vehicles park in on link $(i, j)$, and the other half on $(k, m)$.

\subsection{Feasibility}

As the name weak feasibility suggests, existence of a solution to the flow conservation equations requires a subtler approach because the $p_{i j}$ functions involve make the system of equations nonlinear. 


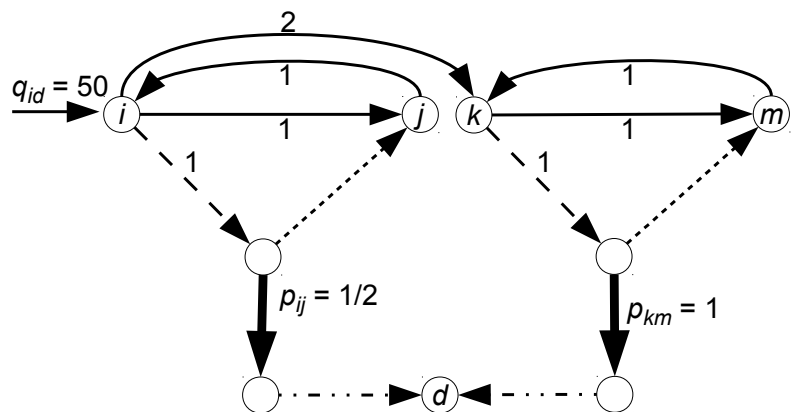

(a)

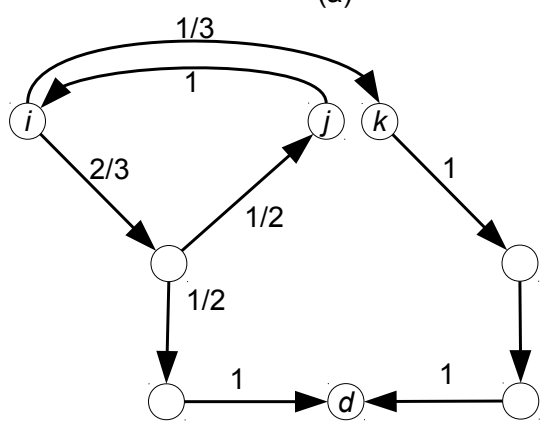

(c)

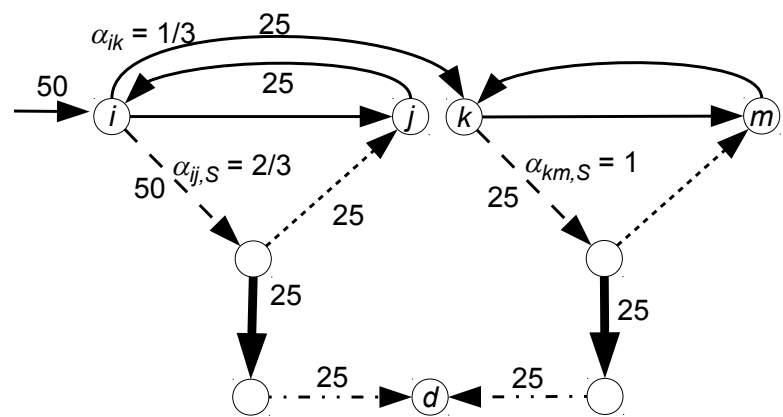

(b)

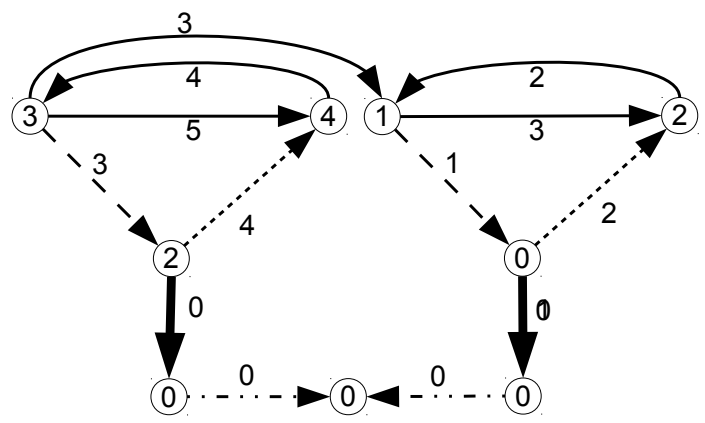

(d)

Figure 2: Example network for demonstration purposes. (a) Problem data, with link travel times and parking probabilities indicated. (b) Network loading ( $\boldsymbol{\alpha}$ and $\mathbf{x}$ ). (c) The induced graph $\hat{G}$. (d) Travel time labels $\boldsymbol{T}$. Non-labeled links have zero values. 


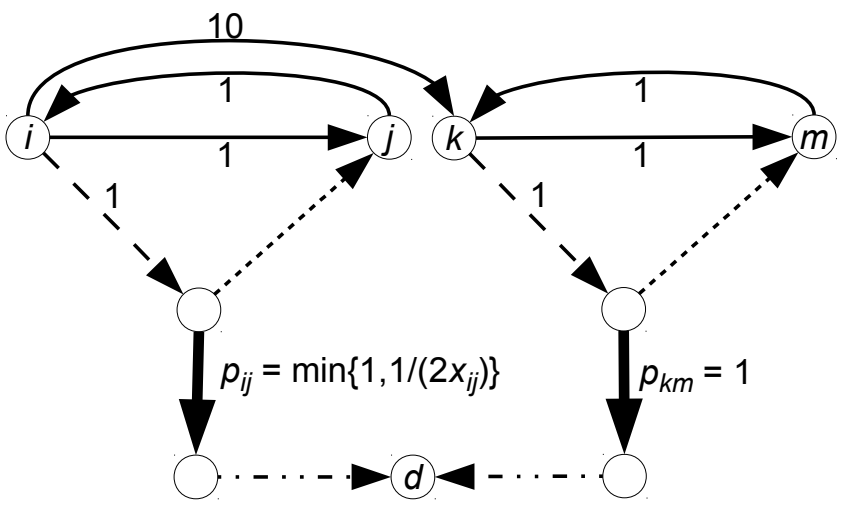

Figure 3: Not all weakly feasible solutions are strongly feasible.

As an example, consider the network in Figure 3, which has a different $p_{i j}$ function than in Figure 2. Let the inflow $q_{i d}$ be $1, \alpha_{i j, S}=\alpha_{j i}=\alpha_{k m, S}=\alpha_{m k}=1$ and all other components of $\boldsymbol{\alpha}$ are zero. The probability of finding parking on link $(i, j)$ is half the reciprocal of the amount of searching flow; this means that the rate at which vehicles park on this link is $f_{i j}\left(x_{i j, S}\right)=1 / 2$ if $x_{i j} \geq 1 / 2$. By contrast, link $(k, m)$ has unlimited parking capacity. The numbers on links indicate costs; links with no adjacent number have zero cost.

Clearly, the given solution is weakly feasible. However, the flow conservation equations for this solution include $x_{i j, S}=1+x_{j i}$ and $x_{j i}=x_{i j, N P}=x_{i j, S}-1 / 2$; by substitution this requires $x_{i j, S}=x_{i j, S}+1 / 2$ which is a contradiction. Intuitively, vehicles are attempting to park on $(i, j)$ at a rate exceeding its capacity and not considering any other options; thus there is no steady-state solution. (There is no issue if the splitting proportions are modified to $\alpha_{i j, S}=\alpha_{i k}=1 / 2$, and all vehicles successfully park.) It is clear that cycling is at the root of possible nonexistence of solutions; in an acyclic network a solution can always be constructed by proceeding in topological order.

Behaviorally, cycles represent the case where a driver returns to a node visited earlier. Traditional equilibrium models exclude this possibility, but modeling parking requires it for several reasons. First, it is common knowledge that drivers searching for on-street parking may "circle the block" several times looking for a convenient space. Second, because parking availability is not deterministic, in general there is no a priori route which ensures an available parking space is found, and nodes may need to be revisited. Third, even when such routes do exist, it is likely that routing policies involving cycles may lead to a lower expected travel times than deterministic ones. If the example in Figure 3 is modified so that $p_{i j} \equiv 1 / 2$ and the original splitting proportions $\alpha_{i j, S}=1$ is used, drivers experience a faster travel time by cycling on $(i, j)$ and $(j, i)$ until a space is found, compared to driving a longer distance to the link $(k, m)$ with guaranteed parking.

The use of constant splitting proportions can be thought of as each driver choosing the next link (and whether to accept an open parking space or not) independent of any such choices in the past, including the current location. This is also consistent with the "sunk cost" interpretation above, but does imply the reset assumption (cf. Provan, 2003; Tang et al., 2014).

Hence, we define a further condition on $\boldsymbol{\alpha}$ called strong feasibility: the vector $\boldsymbol{\alpha}$ is strongly feasible if it is weakly feasible, and if the flow conservation equations have a finite solution in $\mathbf{x}$. Let $\Omega_{S}$ be 
the set of strongly feasible splitting proportions. Below, we characterize this set further and show that under a weak assumption (the functions $f_{i j}(x)$ are strictly increasing and differentiable) the solution to the flow conservation equations is unique when $\boldsymbol{\alpha} \in \Omega_{S}$.

If the parking probabilities $p_{i j}$ were constants, the flow conservation equations would form a linear system and strong feasibility would follow in a simple way from a nonsingularity condition. However, in the more interesting variants of the problem the parking probabilities do depend on the intensity of drivers searching for parking, and in such cases the flow conservation equations form a nonlinear system which is harder to characterize. Particularly, strong feasibility depends not only on the $\boldsymbol{\alpha}$ values themselves but also the parking probability functions. Nevertheless, in this section we provide a few results on the existence of strongly feasible solutions, including a necessary condition and a sufficient condition. The issue of strong feasibility will be discussed further in Section 5.3 on solution algorithms.

Recall that $C_{i j, P}$ was previously defined as the parking capacity of a link. Further define the network capacity $C=\sum_{(i, j)_{P} \in A_{S}} C_{i j, P}$ and the total demand to be $D=\sum_{i \in N} \sum_{d \in N_{D}} q_{i d}$. The section concludes with four results concerning $\Omega_{S}$ : a necessary condition for the existence of a strongly feasible solution, a sufficient condition for the same, a condition for uniqueness of flows corresponding to a strongly feasible solution, and an analytic result which will serve as a lemma for later results.

Proposition 1. (Necessary condition.) If the problem instance (defined by $G$, the p functions, and q) is such that $D>C$, then no strongly feasible solution exists.

Proof. Assume that such an $\boldsymbol{\alpha}$ exists, and let $\mathrm{x}$ be the corresponding feasible solution to the flow conservation equations. By the definition of $C_{i j, P}$, we have $x_{i j, P} \leq C_{i j, P}$ for all $(i, j)_{P}$; summing over all $(i, j)_{P}$ yields

$$
\sum_{(i, j)_{P} \in A_{P}} x_{i j, P} \leq C
$$

Substituting (6) and (8), we have

$$
\sum_{d \in N_{D}} \sum_{i, j) \in A_{T}} x_{i j, T, d}^{d} \leq C
$$

However, by (9) and (10) we have

$$
\sum_{d \in N_{D}} \sum_{(i, j) \in A_{T}} x_{i j, T, d}^{d}=\sum_{d \in N_{D}} \sum_{i \in N_{R}} q_{i d}=D
$$

contradicting $D>C$.

Proposition 2. (Sufficient condition.) If the problem instance satisfies $D<C$, the function $f_{i j}(x)$ is strictly increasing for all $(i, j) \in A$, and the network is strongly connected in the sense that a path exists between any two regular nodes, then at least one strongly feasible solution exists.

Proof. We create such a strongly feasible solution by construction, first identifying $\mathbf{x}$ which satisfy flow conservation and then constructing $\boldsymbol{\alpha}$. Under the stated assumptions $f_{i j}(x)$ is an invertible function on $\left[0, C_{i j, P}\right)$; denote this inverse by $\chi_{i j}$. Choose values $y_{i j}^{h d}$ such that $\sum_{(i, j) \in A} y_{i j}^{h d}=q_{h d}$ 

and $\sum_{h \in N} \sum_{d \in N_{D}} y_{i j}^{h d} \equiv X_{i j}<C_{i j, P}$ (such a choice is always possible when $D<C$; say by solving an assignment problem). Further define $\xi_{i j}=\chi_{i j}\left(X_{i j}\right) / X_{i j}-1$. Now, for each positive $y_{i j}^{h d}$, identify some path $\Pi$ from $h$ to $i$ and some cycle $\Gamma$ from $i$ to itself, only using links in $A_{R}$ (such a path and cycle exist from the assumption of strong connectivity) and define

$$
z_{k \ell}^{h d}=q_{h d}\left([(k, \ell) \in \Pi]+\xi_{i j}[(k, \ell) \in \Gamma]\right)
$$

where the square brackets denote indicator functions using the Iverson notation (equal to one if the quantity in brackets is true, and zero otherwise). Now generate $x_{i j}^{d}=\sum_{h \in N} z_{i j}^{h d}$ for all $d \in N_{D}$, and $x_{i j}$ from (6). This solution satisfies each of the flow conservation equations and is thus strongly feasible.

If $D=C$, then solutions may or may not exist depending on the problem instance.

Proposition 3. If $\boldsymbol{\alpha}$ is strongly feasible and $f_{i j}(x)$ is a strictly increasing function for all links $(i, j)$, then there is exactly one solution $\mathbf{x}$ to the flow conservation equations.

Proof. Assume not, and let $\mathbf{x}$ and $\mathbf{y}$ be two distinct solutions to the flow conservation equations for a given strongly feasible $\boldsymbol{\alpha}$. Without loss of generality choose some link $(i, j)$ and destination $d$ where $x_{i j}^{d}>y_{i j}^{d}$. We can always find such an $(i, j)$ which is a regular link since $x_{i j, T, d}>y_{i j, T, d}$ implies $x_{i j, P}^{d}>y_{i j, P}^{d}$, which (by the assumption that $f_{i j}(x)$ is strictly increasing) implies $x_{i j, S}^{d}>y_{i j, S}^{d}$; equation (7) thus implies $x_{i j}^{d}>y_{i j}^{d}$; a similar argument holds if $x_{i j, N P}^{d}>y_{i j, N P}^{d}$.

Equation (7) thus implies that $x_{h i}^{d}>y_{h i}^{d}$ for some link $(h, i) \in \mathcal{A}$. This argument can be iterated until a complete, finite set of links $\mathcal{A}^{+}(i) \subseteq \mathcal{A}$ has been obtained for which $\left(i^{\prime}, j^{\prime}\right) \in \mathcal{A}^{+}(i)$ implies $x_{i^{\prime} j^{\prime}}^{d}>y_{i^{\prime} j^{\prime}}^{d}$ and for which a path exists from $i^{\prime}$ to $i$ in $G$ with strictly positive $\boldsymbol{\alpha}^{\boldsymbol{d}}$ components for each link in the path. Sum up the flow conservation equations for each link in $\mathcal{A}^{+}(i)$; the resulting equations show that the inflows and outflows must balance. Since $f_{i j}(x)$ is increasing, the difference in outflows $\left(\mathbf{x}^{\mathbf{d}}-\mathbf{y}^{\mathbf{d}}\right)$ on all links (completed trips plus trips routed to other regular or searching links outside of $\mathcal{A}^{+}(i)$ ) must be strictly positive. However, by the completeness of $\mathcal{A}^{+}(i)$, the corresponding terms of $\mathbf{x}-\mathbf{y}$ for incoming links must be nonpositive, which is a contradiction.

If $\boldsymbol{\alpha}_{\mathbf{0}}$ is strongly feasible and $f_{i j}(x)$ is strictly increasing for all links, then the corresponding flows $\mathbf{x}$ are such that $x_{i j, P}$ is strictly less than $C_{i j, P}$ for all $(i, j)_{P}$.

Proposition 4. If $\boldsymbol{\alpha}_{\mathbf{0}}$ is strongly feasible, $x p(x)$ is differentiable, and $\frac{d}{d x} f(x)>0$ for all links, then there exists some neighborhood $\mathcal{B}$ around $\boldsymbol{\alpha}_{\mathbf{0}}$ such that for all $\boldsymbol{\alpha} \in \mathcal{B} \cap \Omega, \boldsymbol{\alpha}$ is strongly feasible.

Proof. Under the hypotheses of the proposition, the Jacobian matrix of the flow conservation equations is continuous and irreducibly diagonally dominant (thus nonsingular) in some neighborhood $\mathcal{B}$ around $\boldsymbol{\alpha}_{\mathbf{0}}$. Then, by the implicit function theorem the function $\mathbf{x}(\boldsymbol{\alpha})$ exists and is continuous in $\mathcal{B}$. Thus, any weakly feasible $\boldsymbol{\alpha}$ in this neighborhood is strongly feasible. 


\subsection{A network loading algorithm}

Consider the "flow-pushing" network loading algorithm, depicted in Algorithm 1, which attempts to find a solution to the flow conservation equations. It uses variables $\eta_{i}^{d}$ to reflect the flow conservation "imbalance" (inflow minus outflow) at each node at the time of processing. In the following algorithm $\eta_{i}$ is used as a shorthand for $\sum_{d \in N_{D}} \eta_{i}^{d}$. Likewise $x_{i j}$ is always understood in the sense of (6) as a shorthand for the sum of the current destination-specific $x_{i j}^{d}$ values.

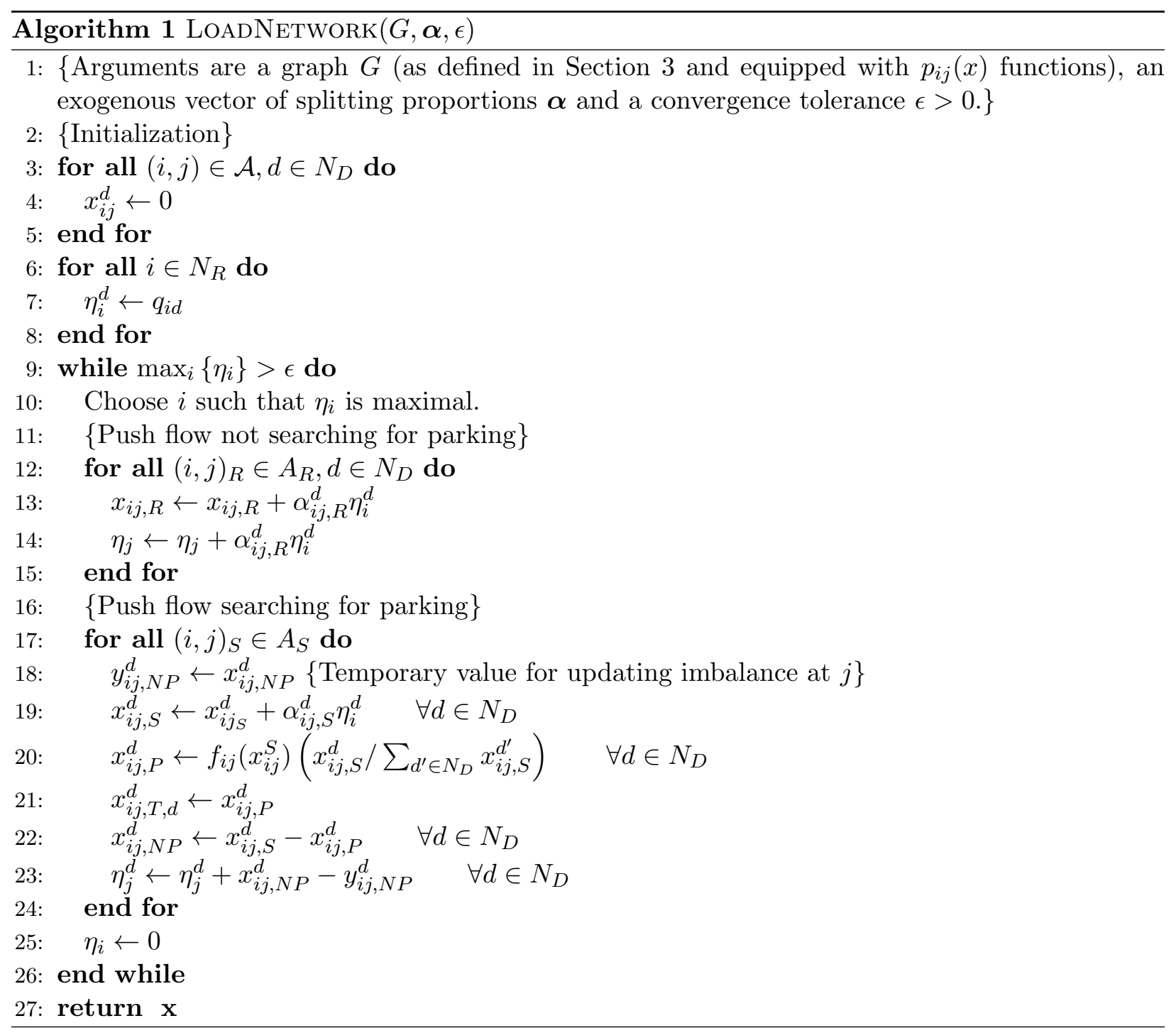

Proposition 5. When line 9 of LOADNETWORK is first executed (and then for the remainder of the algorithm), $\eta_{i} \geq 0$ for all $i$ if all $f_{i j}(x)$ are nondecreasing.

Proof. The result is clearly true when line 9 is executed for the first time. By induction, assume that it is true at some point when line 9 is executed and consider the next steps of the algorithm until line 9 is executed again. The only $\eta$ values which change until line 9 is executed the next time are those selected in lines 14,23 , and 25 . In line $14, \eta_{j}$ is increased by $\alpha_{i j}^{d} \eta_{i}^{d}$ which is nonnegative; 
in line $23, x_{i j, N P}^{d} \geq y_{i j, N P}^{d}$ if $f_{i j}(x)$ is nondecreasing; and line 25 simply sets $\eta_{i}$ to zero. In all cases the induction hypothesis holds.

Proposition 6. Algorithm LOADNETWORK always terminates in finitely many iterations if $\boldsymbol{\alpha}$ is strongly feasible, $f(x)$ is differentiable, and $0<\frac{d}{d x} f(x) \leq 1$ for all links.

Proof. To simply notation, assume there is a single destination; the logic of the proof generalizes naturally to the case of multiple destinations. Define the potential function $U=\sum_{i} \eta_{i}$. Since the $\eta_{i}$ are nonnegative at each step of the algorithm, showing $U \rightarrow 0$ implies $\eta_{i} \rightarrow 0$ for all nodes and that flow conservation equations (7) are satisfied. Equations (1), (2), (8), and (9) are obviously satisfied during the updates "push flow searching for parking." Furthermore, it is not difficult to show that $\sum_{i \in N_{R}} q_{i d}-\sum_{(i, j)_{T} \in A_{T}} d_{i j, T, d}^{d}=\sum_{i} \eta_{i}^{d}$ is an invariant after line 23 is performed, and thus $U=0$ implies (10) is satisfied as well. Since $\boldsymbol{\alpha}$ is strongly feasible and $x p_{i j}(x)$ is strictly increasing, at the unique solution $x^{*}$ to the flow conservation equations we have $0<\left.\delta_{i j} \equiv \frac{d}{d x}\left(f_{i j}\left(x_{i j}^{S}\right)\right)\right|_{x_{i j}^{*}}$

Whenever node $i$ is selected for "pushing," flow potentially increases on all regular and searching links emanating from $i$. Flow pushed onto a regular link $(i, j)$ does not affect $U$ (the imbalance is simply shifted from $i$ to $j$ ), whereas some of the flow pushed onto searching links may end up parking and reaching the destination. Consider one such searching link $(i, j)_{S}$; after performing line 19 the flow $x_{i j, S}^{d}$ increases by $\alpha_{i j, S} \eta_{i}^{d}$. The change in parking flow on $(i, j)_{P}$ is thus

$$
f_{i j}\left(x_{i j}^{S}+\alpha_{i j}^{S} \eta_{i}^{d}\right)-f_{i j}\left(x_{i j}^{S}\right)
$$

which is positive since $f_{i j}(x)$ is strictly increasing. Likewise, the change in flow in $(i, j)_{N P}$ is

$$
x_{i j, N P}-y_{i j}=\alpha_{i j}^{S} \eta_{i}^{d}-\left[f_{i j}\left(x_{i j}^{S}+\alpha_{i j}^{S} \eta_{i}^{d}\right)-f_{i j}\left(x_{i j}^{S}\right)\right]
$$

and it is this amount which is added to $\eta_{j}$. Since $\delta_{i j}<\frac{d}{d x}(f(x)) \leq 1$ we have $0 \leq x_{i j, N P}-y_{i j}<$ $\alpha_{i j}^{S} \eta_{i}\left(1-\delta_{i j}\right)$ by the mean value theorem. Therefore, the increase in $U$ from performing lines 16-23 of the algorithm is no greater than $\eta_{i} \sum_{(i, j)^{S} \in \mathcal{A}} \alpha_{i j}^{S}\left(1-\delta_{i j}\right)$. When step 24 is performed, $U$ decreases by $\eta_{i}$; therefore the total change $\Delta U$ in $U$ from performing steps 10-24 is given by

$$
\Delta U \leq \eta_{i}\left(\sum_{(i, j) \in A} \alpha_{i j}+\sum_{(i, j)^{S} \in A^{S}} \alpha_{i j}^{S}\left(1-\delta_{i j}\right)-1\right)
$$

Since $\sum_{(i, j) \in A} \alpha_{i j}+\sum_{(i, j) \in A^{S}} \alpha_{i j}^{S}=1$ this simplifies to

$$
\Delta U \leq-\eta_{i}\left(\sum_{(i, j)^{S} \in A^{S}} \alpha_{i j}^{S} \delta_{i j}\right)
$$

For any node $i$, let $\bar{\alpha}_{i}=\sum_{(i, j) \in \mathcal{A}} \alpha_{i j}$ denote the total fraction of flow searching for parking on a link leaving node $i$. (In particular, if $\bar{\alpha}_{i}=0$, all vehicles passing through node $i$ are not immediately searching for parking.) Further define $P=\left\{i \in N_{D}: \bar{\alpha}_{i}>0\right\}, \bar{\alpha}=\min _{i \in P} \bar{\alpha}_{i}$, and $\delta=\min _{(i, j) \in A} \delta_{i j}$. 

be the value of $U$ at this point. Whenever $i(k) \notin P$, we have $U_{k+1}=U_{k}$; otherwise

$$
\begin{aligned}
U_{k+1} & =U_{k}+\Delta U \\
& \leq U_{k}-\eta_{i(k)}\left(\sum_{(i(k), j) \in A^{S}} \alpha_{i(k), j}^{S} \delta_{i(k), j}\right) \\
& \leq U_{k}-\frac{U_{k}}{|N|}\left(\sum_{(i(k), j) \in A^{S}} \alpha_{i(k), j}^{S} \delta_{i(k), j}\right) \\
& =U_{k}\left(1-\frac{\bar{\alpha} \delta}{|N|}\right)
\end{aligned}
$$

where the second inequality follows because $i(k)$ is a node with maximal $\eta$ value. Thus $\lim _{k \rightarrow \infty} U_{k}=$ 0 unless $i(k) \in P$ only finitely many times. However, in that case, there is a cycle of nodes which does not send any flow onto searching links and the flow conservation equations cannot be satisfied, contradicting the assumption of strong feasibility.

At termination, the algorithm clearly produces a flow $\mathbf{x}$ which deviates from the flow conservation equations by no more than $\epsilon$ for each link. A trivial corollary is that if the potential function $U$ does not convege linearly to zero, then the given solution is not strongly feasible. This can be used as a strong feasibility test, much in the same way that nonconvergence of label-correcting shortest path algorithms can be used to test for existence of negative-cost cycles.

\subsection{Interpretation from a driver's perspective}

The parking model described in this paper is formulated in terms of aggregate flows $\mathbf{x}$. However, this model can be viewed through the perspective of an individual driver as well. This section formulates this as a Markov chain, and shows that if the aggregate flows $\mathbf{x}$ are induced by a strongly feasible $\boldsymbol{\alpha}$, then the probability that each driver reaches his or her destination asymptotically approaches 1 after a sufficient amount of driving and searching time.

In this subsection, consider an individual driver who departs origin node $o \in N_{R}$ destined for node $d \in N_{D}$ (thus $q_{o d}>0$ ). Also assume that the splitting proportions $\boldsymbol{\alpha}$ are strongly feasible, so the aggregate flow vector $\mathbf{x}$ is well-defined and uniquely determined. The progression of this driver through the network can be expressed as a Markov chain, where the states are the set of all nodes $\mathcal{N}$, the initial state is $o$, and the transition probabilities are given as follows: for each regular node $i \in N_{R}$ and outgoing link $(i, j) \in \mathcal{A}$, the probability of transitioning from $i$ to $j$ is given by $\alpha_{i j}$. For each parking node $i j_{P}$ corresponding to link $(i, j)$, the probability of transitioning from $i j_{P}$ to the corresponding transfer node $i j_{T}$ is $p_{i j}$ and to the regular node $j$ is $1-p_{i j}$. For each transfer node, the transition probability to the destination $d$ is 1 , and 0 for all other nodes. The destination node $d$ is an absorbing state.

We aim to show that if $\boldsymbol{\alpha}$ is strongly feasible, the Markov chain reaches $d$ with probability asymptotically reaching 1 . To assist with this, define the set $\hat{\mathcal{N}}$ denoting all states of the Markov chain reached with positive probability when starting from $o$. More formally, $\hat{\mathcal{N}}$ is defined as the smallest 

asymptotic result is obtained in two steps, first showing that strong feasibility of $\boldsymbol{\alpha}$ implies that the destination $d$ is part of the reachable set $\hat{\mathcal{N}}$, then showing that this probability approaches 1 as the driver spends longer searching.

Proposition 7. If $\boldsymbol{\alpha}$ is strongly feasible, then $d \in \hat{\mathcal{N}}$.

Proof. The flow conservation equations above are given in terms of links; however together with weak feasibility they imply the following relations for nodes in $N_{R}, N_{P}, N_{T}$, and $N_{D}$, respectively:

$$
\begin{aligned}
q_{i d}+\sum_{(h, i) \in \mathcal{A}} x_{h i}^{d}=\sum_{(i, j) \in \mathcal{A}} x_{i j}^{d} & \forall i \in N_{R}, d \in N_{D} \\
x_{i j, S}^{d}=x_{i j, P}^{d}+x_{i j, N P}^{d} & \forall(i, j)_{S} \in A_{S}, d \in N_{D} \\
x_{i j, P}^{d}=x_{i j, T, d}^{d} & \forall(i, j)_{P} \in A_{P}, d \in N_{D} \\
\sum_{(i, j)_{T}^{d} \in A_{T}} x_{i j, T, d}^{d}=\sum_{k \in N_{R}} q_{k d} & \forall d \in N_{D}
\end{aligned}
$$

The left-hand side of each equation represents the "inflow" to a node, while the right-hand side represents the "outflow." Summing these equations over a subset of nodes thus equates the total inflow to the subset to the total outflow. The flow $x_{i j}^{d}$ on links connecting two nodes of such a subset appears on both sides, and thus can be canceled. By definition of $\hat{\mathcal{N}}$, in such a sum, no positive $x_{i j}^{d}$ appears on the right-hand side without also appearing on the left-hand side. We thus have

$$
\sum_{i \in N_{R} \cap \hat{\mathcal{N}}} q_{i d}+\sum_{(i, j) \in \mathcal{A}: i \notin \hat{\mathcal{N}}} x_{i j}^{d}=[d \in \hat{\mathcal{N}}] \sum_{i \in N_{R}} q_{i d}
$$

again using the bracket notation for the indicator function. Since $q_{o d}>0$, the left-hand side of the equation is positive. Strong feasibility implies that this equation is consistent and thus the right-hand side must be positive as well; this is only possible if $d \in \hat{\mathcal{N}}$.

Proposition 8. Let $P(t)$ be the probability that the (absorbing) destination state d has been reached after $t$ transitions from the initial state o, with transition probabilities based on a given $\boldsymbol{\alpha}$. If $\boldsymbol{\alpha}$ is strongly feasible, then $\lim _{t \rightarrow \infty} P(t)=1$.

Proof. Let $\hat{G}$ be the subgraph of $G$ induced by the node set $\hat{\mathcal{N}}$. (See Figure 2(c), with transition probabilities for the Markov chain indicated on the links.) By Proposition 7 and the definition of $\hat{\mathcal{N}}$ there is at least one simple path in $\hat{G}$ from each node $i \in \hat{\mathcal{N}}$ to the destination $d$, and the probability of successivly transitioning from state $i$ to $d$ by traversing this path is given by the product of the transition probabilities along successive arcs (the relevant $\alpha$ value for regular or searching links, and $p$ or $1-p$ for parking and no parking links, respectively). Again by definition of $\hat{\mathcal{N}}$ at least one such path has strictly positive traversal probability. Let $K$ be the maximum length of such a simple path, and let $\hat{p}>0$ be the least traversal probability of any such path. Regardless of the initial state, after $K$ transitions we have $P(K) \geq \hat{p}$ since the destination may be reachable via multiple paths in $\hat{G}$. After $n K$ transitions, we have $P(n K)=1-(1-P(n K)) \geq 1-(1-\hat{p})^{n}$ with the inequality following because every $K$ steps the probability of reaching $d$ is at least $\hat{p}$. Since $d$ is absorbing, $P(t)$ is nondecreasing and $P(t) \geq P(K\lfloor t / K\rfloor) \geq 1-(1-\hat{p})^{\lfloor t / K\rfloor}$ which asymptotically approaches 1 when $t$ is large. 


\section{Equilibium}

This section introduces the equilibrium framework built upon the network loading from Section 4 . Its major goals are (1) expressing the expected travel time to the destination as a function of the choices made at each node; (2) developing a preliminary variational inequality formulation of the equilibrium problem; (3) establishing a more practical approach to feasibility than strong feasibility, and its suitability for equilibrium; and finally (4) a convex combinations heuristic for solving the equilibrium problem.

\subsection{Cost labels}

As a first step toward development of the equilibrium model, we calculate the costs associated with each choice travelers can make. These cost labels play an analogous role to the value functions in dynamic programming, reflecting the expected remaining travel cost between the current node and the destination, including any driving time costs, parking costs, walking time, and additional time due to searching. These calculations are made separately from the network loading, so we can assume that a flow vector $\mathbf{x}$ satisfying the flow conservation equations is given and held constant. For each destination $d$ and regular link $(i, j)_{R}$, let $T_{i j, R}^{d}$ represent the expected remaining cost among travelers from node $i$ to the destination $d$, including the cost on link $(i, j)_{R}$ and remaining costs to the destination:

$$
T_{i j, R}^{d}=t_{i j, R}+\sum_{(j, k) \in A_{R} \cup A_{S}} \alpha_{j k}^{d} T_{j k}^{d}
$$

where $T_{j k}^{d}$ reflect downstream costs from $d$. For searching links, the corresponding labels are defined as:

$$
T_{i j, S}^{d}=t_{i j, S}+p_{i j}\left(t_{i j, P}+t_{i j, T, d}\right)+\left(1-p_{i j}\right)\left(t_{i j, P}+\sum_{(j, k) \in A_{R} \cup A_{S}} \alpha_{j k}^{d} T_{j k}^{d}\right)
$$

As a demonstration of this concept, Figure 2(d) shows the $\boldsymbol{T}$ labels for the example network and network loading shown in previous panels of that figure.

Note that $p_{i j}$ can be treated as constant in (21) since $\mathbf{x}$ is given and fixed. Hence (20) and (21) form a linear system in $T_{i j}^{d}$ and $T_{i j, S}^{d}$ with $\left|N_{D}\right|\left(\left|A_{R}\right|+\left|A_{S}\right|\right)$ equations and variables. While this linear system can be solved directly and without excessive difficulty (since transportation networks are relatively sparse), a network algorithm similar to LOADNETWORK performs even better. This algorithm, CalculateCostlabels is presented as Algorithm 2. In this algorithm, $\boldsymbol{\alpha}$ and $\boldsymbol{p}$ are to be calculated from the flow vector $\mathbf{x}$ provided as an argument. Given a vector $\mathbf{T}$ of cost labels which do not necessarily solve (20) and (21), the "imbalance" $\zeta_{i}$ of node $i$ is calculated by summing the absolute difference between the left and right hand sides of (20) and (21) across all outgoing links and destinations:

$$
\zeta_{i}=\sum_{d}\left(\sum_{(i, j)_{R} \in A_{R}}|\operatorname{RHS}(20)-\operatorname{LHS}(20)|+\sum_{(i, j)_{S} \in A_{S}}|\operatorname{RHS}(21)-\operatorname{LHS}(21)|\right)
$$

At each iteration a node $i$ with maximum $\zeta_{i}$ is chosen, and the labels $T_{i j}^{d}$ of outgoing links are calculated using (20) and (21). Convergence of this algorithm is not difficult to show, since each 

as least as much as the Gauss-Seidel method, which is sure to converge since the linear system is irreducibly diagonally dominant (as follows from strong feasibility).

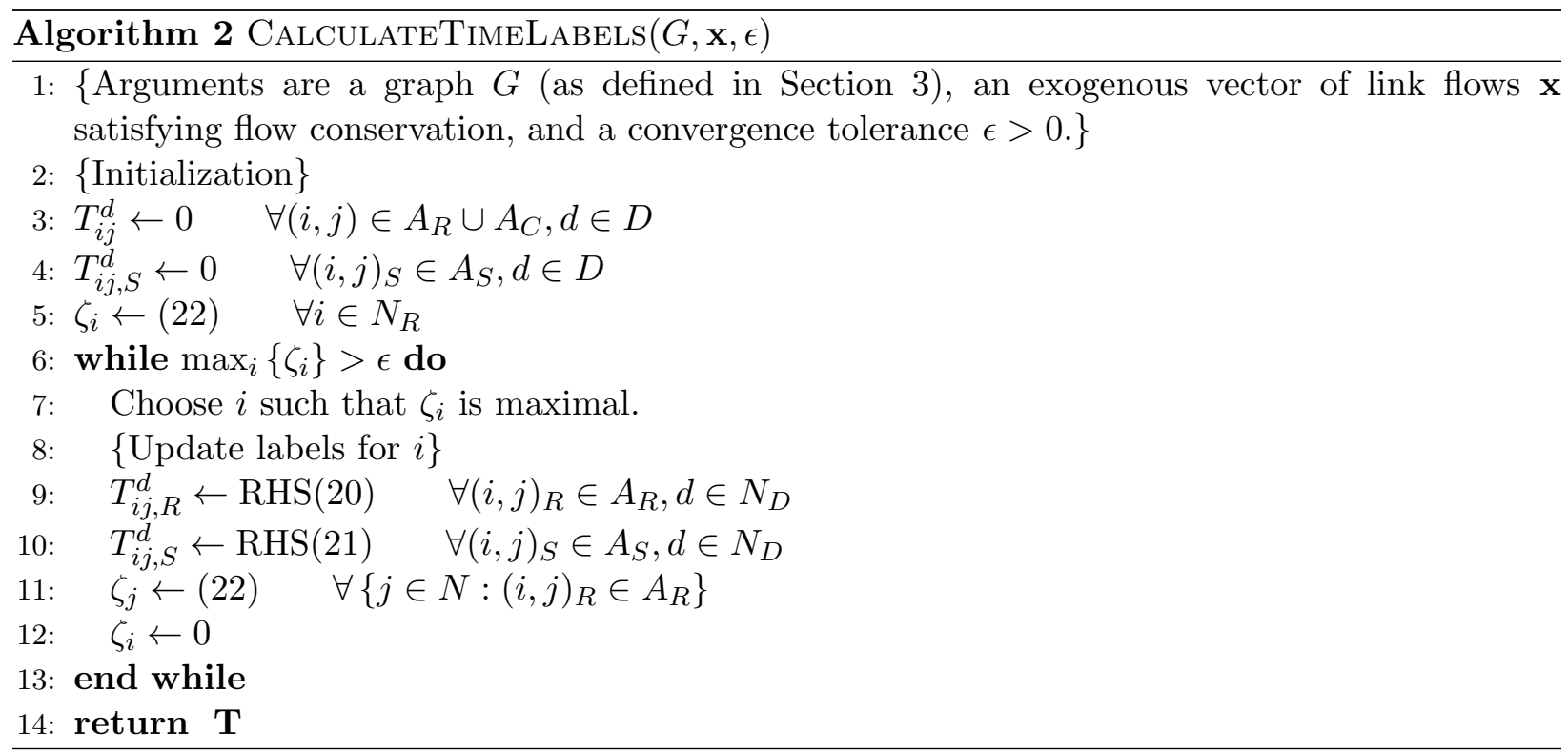

\subsection{Equilibrium formulation}

The cost labels defined in the previous subsection lead to formulating an equilibrium principle, that a given alternative will be used only if its cost is minimal among all alternatives (implying equality of cost when multiple alternatives are used). Let $\boldsymbol{\alpha}$ be strongly feasible; then there is a unique network loading $\mathbf{x}(\boldsymbol{\alpha})$ based on the flow conservation equations, and a unique set of travel time labels $\mathbf{T}(\mathbf{x}(\boldsymbol{\alpha}))$ based on (20) and (21); for brevity, this latter relation is abbreviated $\mathbf{T}(\boldsymbol{\alpha})$.

A strictly positive $\alpha_{i j}^{d}$ value suggests that some travelers heading to $d$ and passing through node $i$ are leaving via node $j$; at equilibrium this is only possible if $T_{i j}^{d}$ is no greater than $T_{i j^{\prime}}^{d}$ for any other outgoing link $\left(i, j^{\prime}\right)$. That is, a strictly feasible $\boldsymbol{\alpha}$ is defined to be an equilibrium if

$$
\alpha_{i j}^{d}>0 \Rightarrow T_{i j}^{d}=\min _{\left(i, j^{\prime}\right) \in A_{R} \cup A_{S}}\left\{T_{i j^{\prime}}^{d}\right\}
$$

is satisfied for all $(i, j)$ and $d$. The reader may verify that Figure 2 portrays an equilibrium solution.

Proposition 9. If $f(x)$ is differentiable and $\frac{d}{d x} f(x)>0$, then a strongly feasible vector of splitting proportions $\boldsymbol{\alpha}^{*}$ is an equilibrium if $\mathbf{T}\left(\boldsymbol{\alpha}^{*}\right) \cdot\left(\boldsymbol{\alpha}^{*}-\boldsymbol{\alpha}\right) \leq 0$ for all $\boldsymbol{\alpha} \in \Omega_{S}$.

Proof. Assume that $\boldsymbol{\alpha}^{*}$ is not an equilibrium. Then there exist two distinct links $(i, j) \in \mathcal{A}$ and $\left(i^{\prime}, j^{\prime}\right) \in \mathcal{A}$ and some $d \in N_{D}$ such that $\alpha_{i j}^{* d}>0$ and $T_{i j}^{d}<T_{i^{\prime} j^{\prime}}^{d}$. By Proposition 4, the vector $\boldsymbol{\alpha}=\boldsymbol{\alpha}^{*}+\delta \boldsymbol{\Delta}$ is strongly feasible for sufficiently small positive $\delta$, where $\boldsymbol{\Delta}$ has two nonzero components: +1 for $\left(i^{\prime}, j^{\prime}\right)$ and $d$, and -1 for $(i, j)$ and $d$; in this case $\mathbf{T}\left(\boldsymbol{\alpha}^{*}\right) \cdot \boldsymbol{\alpha}^{*}>\mathbf{T}\left(\boldsymbol{\alpha}^{*}\right) \cdot \boldsymbol{\alpha}$ and the variational inequality is not satisfied. 
Unfortunately, the utility of this formulation is limited by the irregular structure of $\Omega_{S}$ : Proposition 4 suggests that $\Omega_{S}$ is not a closed set, unless it includes the entire boundary of $\Omega$; and in any case, $\Omega_{S}$ need not be convex. This is demonstrated in Figure 4, where the left and right parking links have capacity 5 , the center parking link has capacity 1 , and the total demand is 4 . Panel (a) shows the network with relevant parameters (link styles are the same as in Figure 1), and panels (b) and (c) respectively illustrate two strongly feasible solutions in which all vehicles park either at the left or right links. Panel (d) illustrates the average of these two strongly feasible solutions; in this solution, 1 vehicle attempts to park at the left link, 1 vehicle attempts to park at the right link, and 2 vehicles attempt to park at the middle link. The number of vehicles attempting to park at the middle link exceeds its capacity, and the network structure forces drivers to continue searching on the link they initially chose, so this solution is not strongly feasible.

These properties of $\Omega_{S}$ are not favorable. The vast majority of variational inequality results and algorithms, including fixed-point theorems to establish equilibrium existence, require the feasible set to be closed and convex (cf. Facchinei and Pang, 2003). To address this, the following subsection describes a modification to the network structure and feasible set which are more useful.

\subsection{A practical approach to feasibility}

As shown in the previous section, $\Omega_{S}$ need not be a closed or convex set, which poses difficulties both for demonstrating existence of an equilibrium solution and for finding such a solution if it exists. Since $\Omega$ is closed convex, the difficulty is introduced by requiring a finite solution to the flow conservation equations. An alternative method is to transform the network further by adding a direct link from each node $i$ to each destination $d$; let each such link have a high, but finite travel time $\bar{t}$. Denote the collection of these links $\bar{A}$, and the new network $\bar{G}$. Given a small positive parameter $\epsilon$, define the set $\Omega_{\epsilon}=\left\{\alpha \in \Omega: \alpha_{i j} \geq \epsilon \quad \forall(i, j) \in \bar{A}\right\}$.

Intuitively, the new links in $\bar{A}$ create direct connections to the destination with high cost. One possible behavioral interpretation is that these links represent "failed trips" where travelers give up searching for parking; the connection to the destination is only enforced to maintain flow conservation. Whenever travelers pass through a node, a fraction $\epsilon$ of the drivers will give up searching and terminate their trips. This explanation is not perfect (since the vehicles disappear from the network at this point in time), but facilitates the solution process - as shown below, any set $\Omega_{\epsilon}$ is closed convex and admits a unique solution to the flow conservation equations. Furthermore, link flows $\mathbf{x}$ and travel time labels $\mathbf{T}$ corresponding to $\boldsymbol{\alpha} \in \Omega_{S}$ in $G$ can be approximated arbitrary closely by splitting proportions in $\Omega_{\epsilon}$ and $\bar{G}$ as $\epsilon \rightarrow 0$.

Proposition 10. Given any $\epsilon>0$ and $\boldsymbol{\alpha} \in \Omega_{\epsilon}$, at least one solution exists to the flow conservation equations in $\bar{G}$.

Proof. Apply algorithm LOADPOLICY. When processing node $i$, at least $\epsilon \eta_{i}$ units of flow reach the destination; since $i$ is the node with maximal $\eta_{i}$ value, the reduction in the potential function $U$ is at least $\epsilon U /|N|$ (even neglecting any flow which may find parking), and the algorithm converges linearly to a solution of the flow conservation equations.

Proposition 11. Given any $\epsilon>0$ and $\boldsymbol{\alpha} \in \Omega_{\epsilon}$, the solution to the flow conservation equations in $\bar{G}$ is unique. 


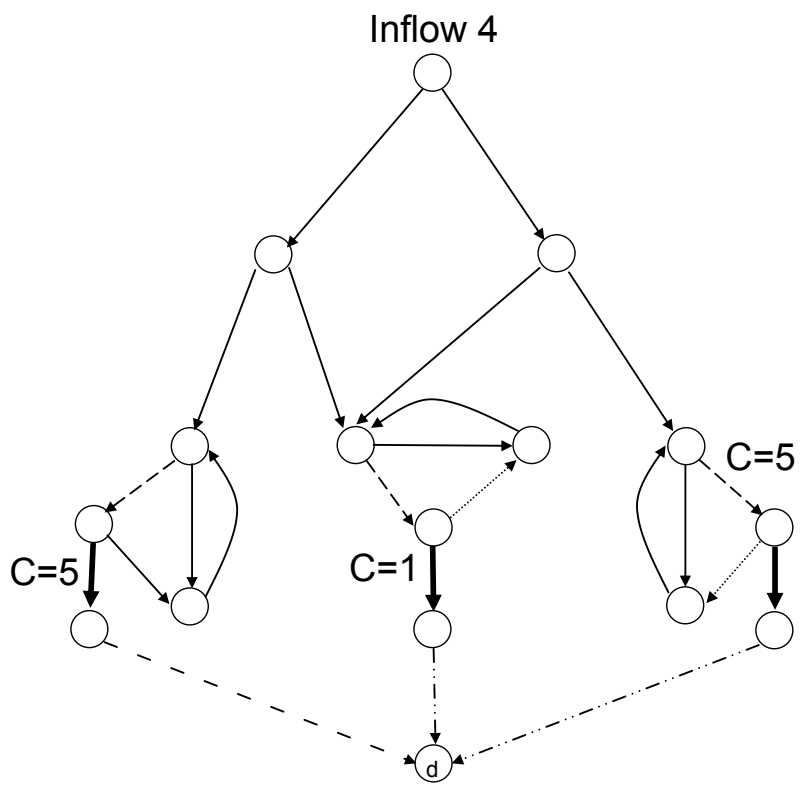

(a)

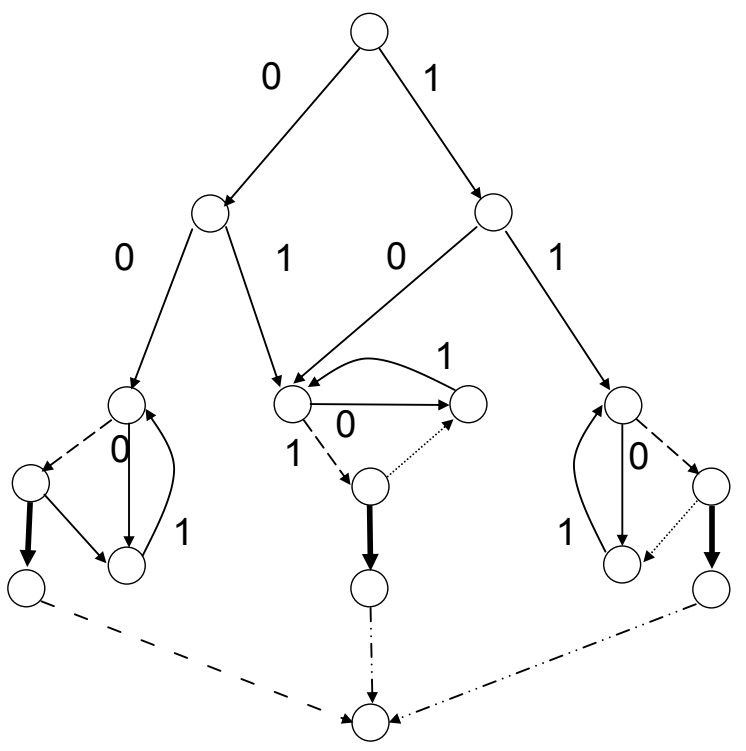

(c)

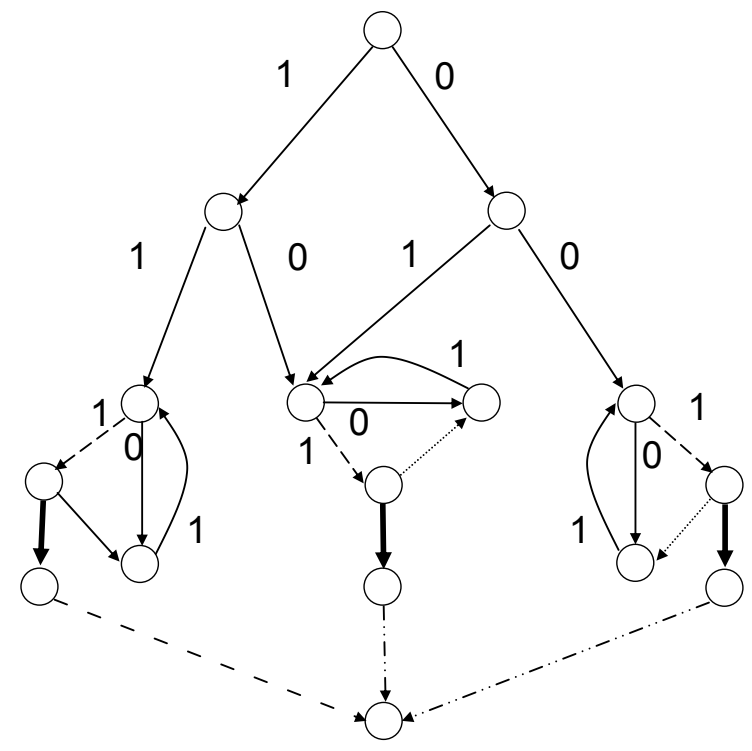

(b)

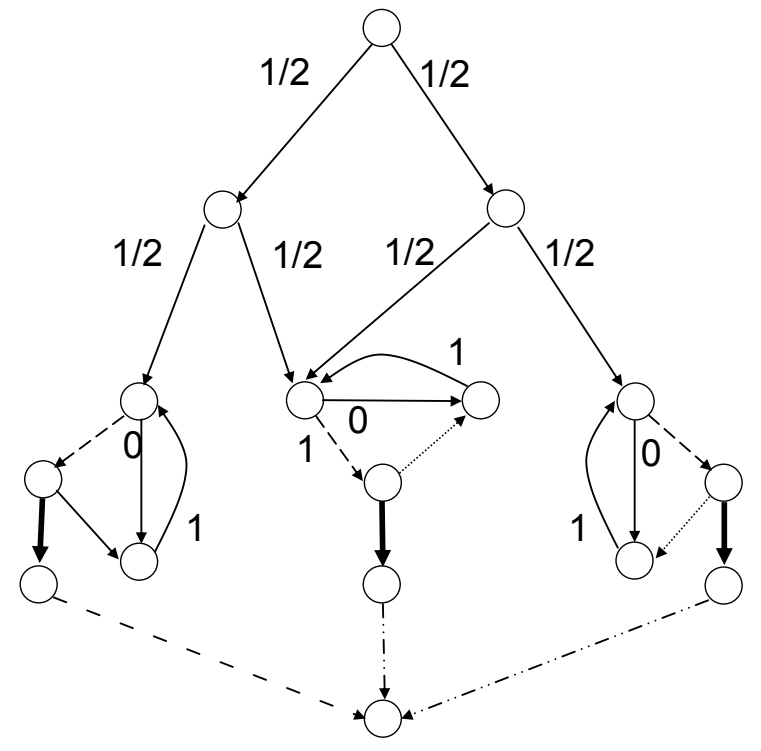

(d)

Figure 4: The set $\Omega_{S}$ is not convex. 
Proposition 12. Let $\mathbf{T}_{\mathbf{0}}$ and $\mathbf{x}_{\mathbf{0}}$ correspond to $\boldsymbol{\alpha}_{\mathbf{0}} \in \Omega_{S}$ in $G$. For any $\delta>0$, there exists an $\epsilon>0$ and $\mathbf{T}_{\epsilon}$ and $\mathbf{x}_{\epsilon}$ corresponding to some $\boldsymbol{\alpha}_{\boldsymbol{\epsilon}} \in \Omega_{\epsilon}$ in $\bar{G}$ such that $\left\|\mathbf{T}_{\epsilon}-\mathbf{T}_{\mathbf{0}}\right\|<\delta$ and $\left\|\mathbf{x}_{\epsilon}-\mathbf{x}_{\mathbf{0}}\right\|<\delta$.

Proof. Define the projection operator $\Pi(\mathbf{z}, K)$ returning the point $\mathbf{z}^{*}$ in $K$ minimizing $\left\|\mathbf{z}^{*}-\mathbf{z}\right\|$; this point is unique if $K$ is convex. Consider some sequence $\left\{\epsilon_{k}\right\}$ converging to zero, and let $\boldsymbol{\alpha}_{\boldsymbol{k}}=\Pi\left(\boldsymbol{\alpha}_{\mathbf{0}}, \Omega_{\epsilon_{k}}\right)$. Clearly as $k \rightarrow \infty,\left\|\boldsymbol{\alpha}_{\boldsymbol{k}}-\boldsymbol{\alpha}_{\mathbf{0}}\right\| \rightarrow 0$. By the implicit function theorem, the function $\mathbf{x}(\boldsymbol{\alpha})$ is continuous when $\boldsymbol{\alpha}$ is strongly feasible, so as $\boldsymbol{\alpha}_{\mathbf{k}} \rightarrow \boldsymbol{\alpha}_{\mathbf{0}}$ we have $\mathbf{x}_{\boldsymbol{\epsilon}} \equiv \mathbf{X}\left(\boldsymbol{\alpha}_{\boldsymbol{k}}\right) \rightarrow \mathbf{x}_{\mathbf{0}}$. Finally, since $\mathbf{T}$ is obtained from $\mathbf{x}$ by solving a nonsingular linear system, the mapping $\mathbf{T}(\mathbf{x})$ is continuous and $\mathbf{T}_{\epsilon} \equiv \mathbf{T}\left(\boldsymbol{\alpha}_{\mathbf{k}}\right) \rightarrow \mathbf{T}_{\mathbf{0}}$.

Proposition 13. For any $\epsilon>0$, there exists at least one equilibrium solution in $\Omega_{\epsilon}$.

Proof. The variational inequality $\mathbf{T}\left(\boldsymbol{\alpha}^{*}\right) \cdot\left(\boldsymbol{\alpha}^{*}-\boldsymbol{\alpha}\right) \leq 0$ for all $\boldsymbol{\alpha} \in \Omega_{\epsilon}$ is defined on a closed, convex set. Furthermore the mapping $\mathbf{T}$ is continuous in $\alpha$ as shown in the proof of Proposition 12. Theorem 2.1.1 of Facchinei and Pang (2003) thus applies and the variational inequality has at least one solution.

\subsection{An equilibrium heuristic}

Although $\mathbf{T}$ is a continuous function of $\boldsymbol{\alpha}$ on $\Omega_{\epsilon}$, ensuring existence of an equilibrium solution, the function lacks other favorable properties (such as monotonicity). Nevertheless, a convex combinations heuristic seems to work well; this is shown in Algorithm 3. This algorithm involves iteratively performing a network loading, calculating travel time labels, identifying a "target" flow proportions vector $\boldsymbol{\alpha}^{*}$ placing maximal weight on minimum-cost choices for each node and destination, and updating the flow proportions by taking a convex combination of the current and target vectors.

Convergence is determined with a gap function measuring deviation from the equilibrium principle. One such gap function is the average excess cost

$$
A E C=\frac{\sum_{i \in N} \sum_{d \in N_{D}} \sum_{(i, j) \in A \cup A_{S}}\left(T_{i j}^{d}-\min _{\left(i, j^{\prime}\right) \in A_{R} \cup A_{S}} T_{i j^{\prime}}^{d}\right)}{D}
$$

As shown in the demonstrations below, the simple choice of $\lambda=1 / k$ appears to work satisfactorally, where $k$ is the number of times algorithm LOADNETwORK has been performed when step 8 is executed.

\section{Demonstration}

The model is demonstrated using the well-known Sioux Falls network (Bar-Gera, 2014) with 24 nodes, 76 links, and 24 origins and destinations. An instance of the parking search equilibrium problem was generated from this network using the following procedure. First, a destination was created for each zone centroid; the walking time from any link to each destination is proportional 


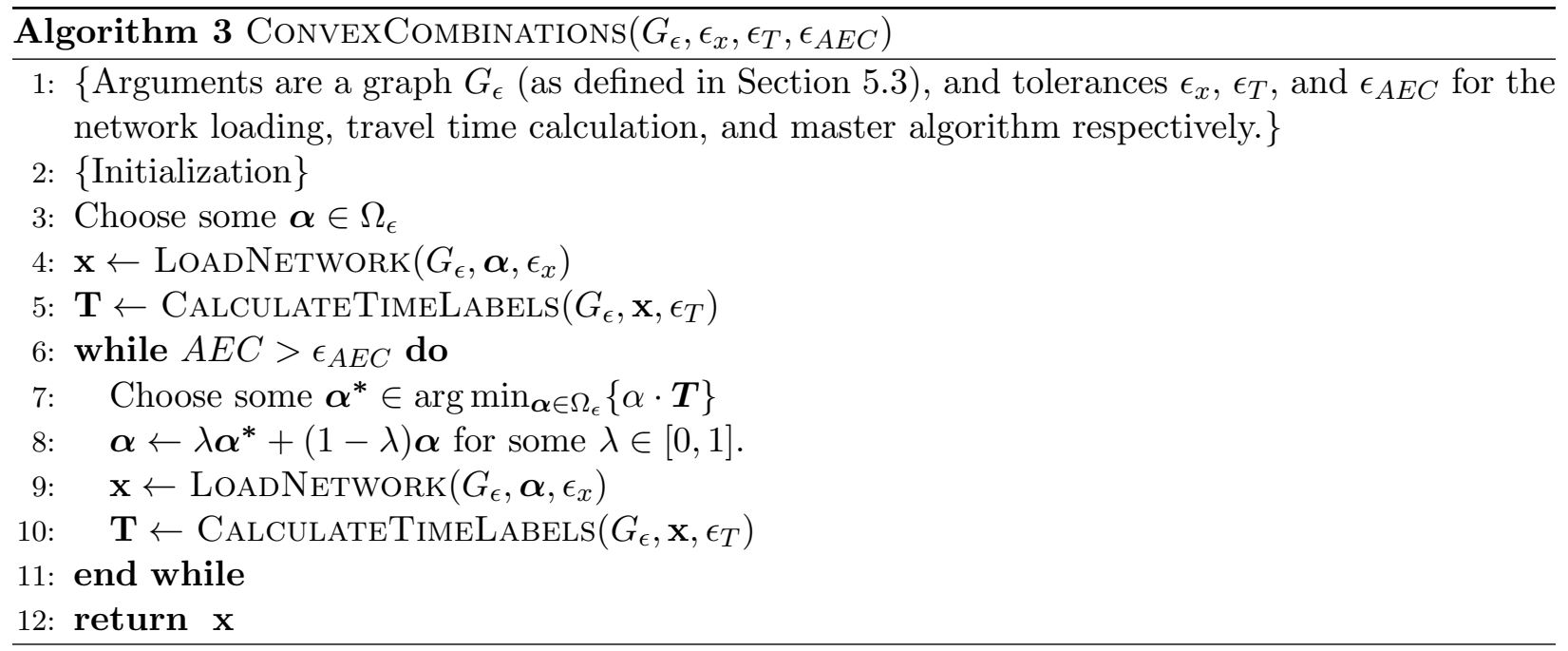

to the Euclidean distance between the origin and destination nodes, using the node coordinates associated with the Sioux Falls network. We assume parking is allowed on each link in the network, with the number of spaces proportional to the physical length of the link. The mean duration of parking and parking cost are uniform on all links.

The algorithms were implemented in $\mathrm{C}$ and run on a $2.60 \mathrm{GHz}$ Intel machine with $4 \mathrm{~GB}$ memory running Windows 7 . Figure 5 shows the convergence rate of the algorithm, reporting the average excess cost obtained after a given amount of computation time has elapsed. The convex combinations method with step size inversely proportional to iteration count seems to function acceptably, at least in this small network, and the gap function decreases steadily as the solution stabilizes.

Figure 6 shows the importance of considering parking in the assignment and equilibrium process. The left panel of the figure shows the link volumes when parking search is neglected ${ }^{1}$ and trips can park at any location with probability 1 (setting $p_{i j}(x) \equiv 1$ in the algorithms). The right panel shows link volumes after accounting for parking search; there is a significant increase in these volumes. Network-wide, the total vehicle-hours traveled (VHT) increased from 24310 to 29059, an increase of 19.5\%. Figure 7 shows the fraction of flow on each link searching for parking (as opposed to driving through) and probability of finding parking at different links. In particular, notice how available parking is scarcer in the city center, and more plentiful around the network perimeter. This model thus provides quantitative estimates of parking availability in different regions of the network.

Two sensitivity analyses were conducted to the input parameters. In the first, the mean parking duration is varied, perhaps reflecting changes in parking time limits. As shown in Figure 8, increasing the duration of parking results in increases in both average driving time (as vehicles search longer before finding an available space) and average walking time (as some drivers accept parking spaces further from their destination). These trends are roughly convex, indicating greater sensitivity to parking duration when parking is scarcer than when it is more plentiful. The second sensitivity analysis varies the relative cost of walking time to driving time, perhaps reflecting changes due to weather (in poor weather, drivers may prefer to spend more time searching inside the vehicle and

\footnotetext{
${ }^{1}$ All links in the Sioux Falls network have a "mirror" with the tail and head node reversed; for clarity in the figures, the higher value of the two mirror links is shown.
} 


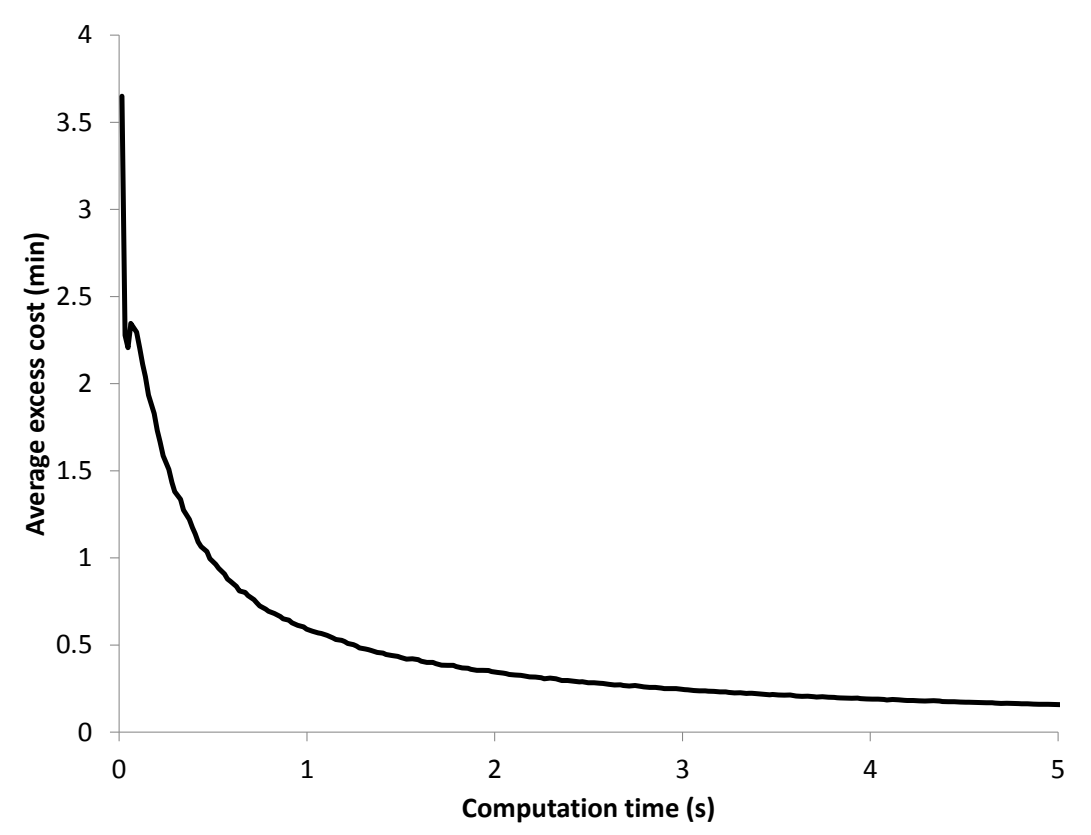

Figure 5: Progress of the convex combinations heuristic

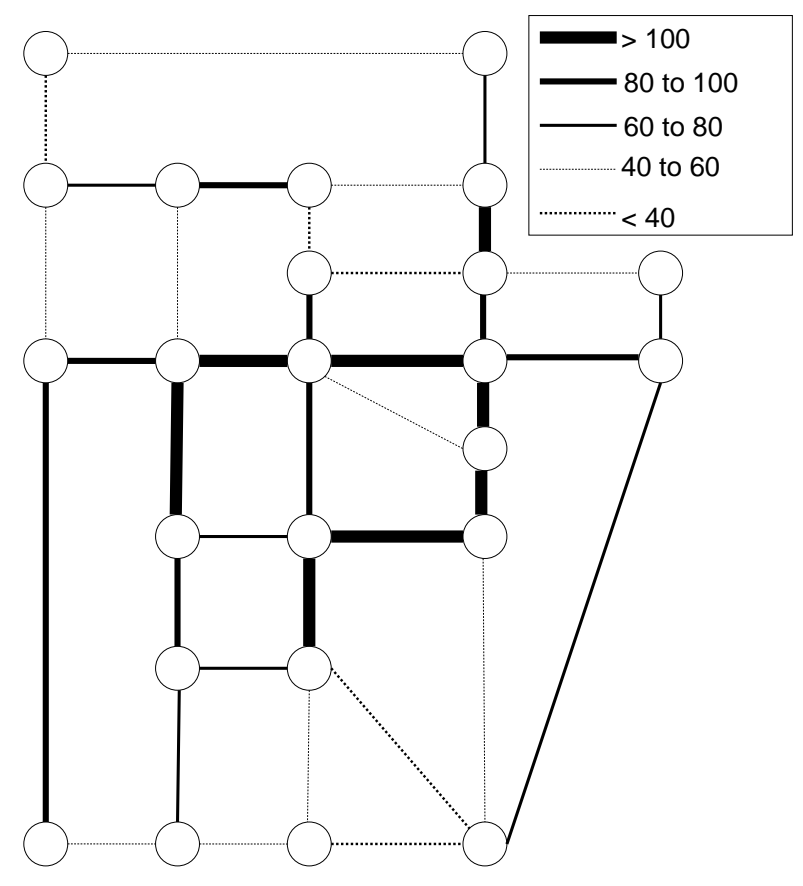

(a)

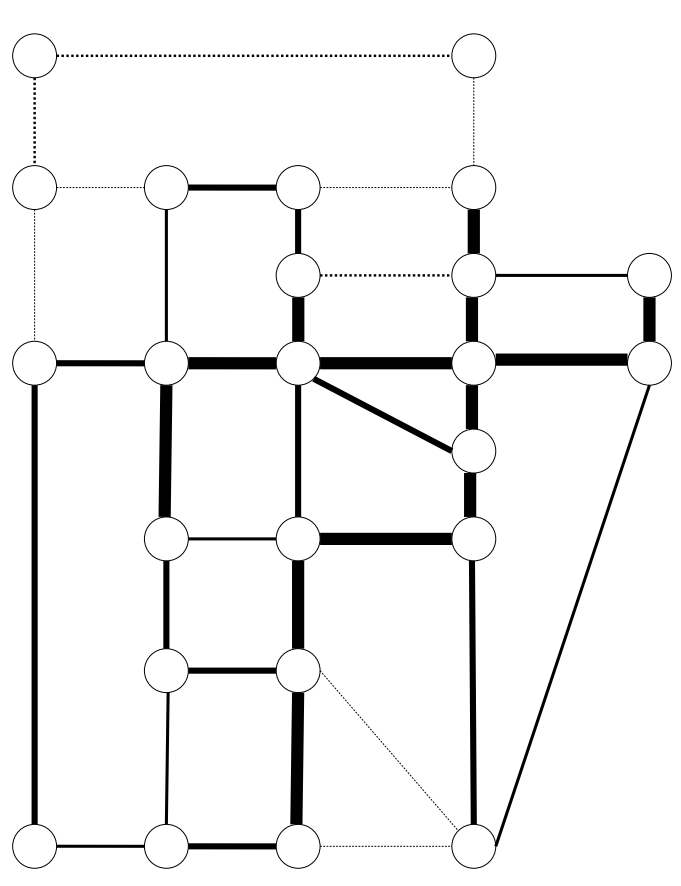

(b)

Figure 6: Traffic volumes (a) without considering parking search and (b) considering search 


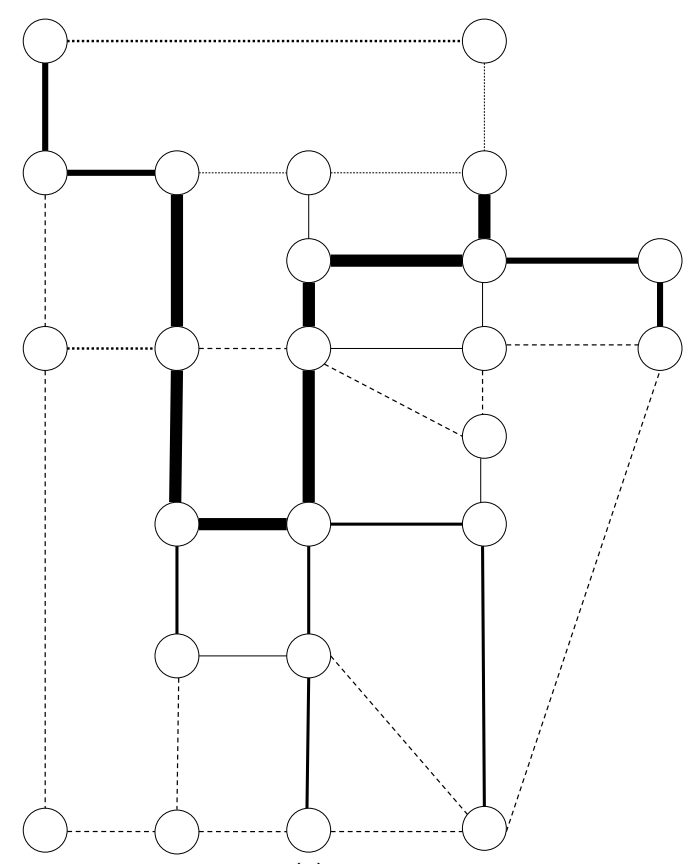

(a)

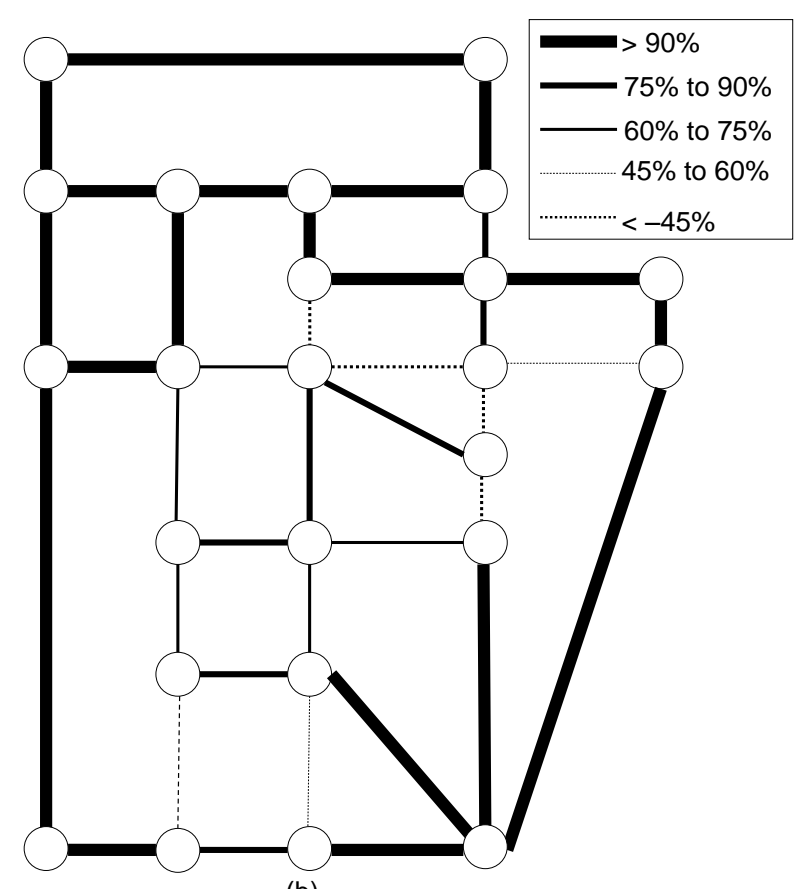

(b)

Figure 7: (a) Fraction of link volume searching for parking and (b) probability of successfully finding parking

less time walking). (Figure 9.) As walking becomes onerous, the amount of driving time increases and the average amount of walking time decreases, but only slightly; this suggests that in the base conditions, drivers are already parking relatively close to their destinations.

\section{Conclusions}

This paper presented an equilibrium model for the parking search process. Notable features of the model are its ability to model the probability of parking availability as a function of searching intensity; the introduction of an equilibrium framework to account for the dependence of searching intensity on parking availability probabilities; and a formulation admitting general networks, allowing the increases in flow on specific links due to parking search to be seen. Computational analysis conducted on the Sioux Falls network showed that accounting for parking led to a nearly 20\% increase in predicted vehicle-hours traveled. The model also quantitatively estimated the spatial difference in parking flows across the network. As vehicles parked longer, both driving and walking time increased reflecting that vehicles were parked further away from their final destinations. Vehicles also spent more time searching for parking under difficult walking conditions.

As the focus of this paper was on formulating the basic model, it would be fruitful to search for more efficient algorithms or algorithms which provably converge to equilibrium, or to more fully develop practical case studies using field data on parking availability and destinations. The model itself can also be extended to account for congestion in link travel times, dynamic evolution in demand and 


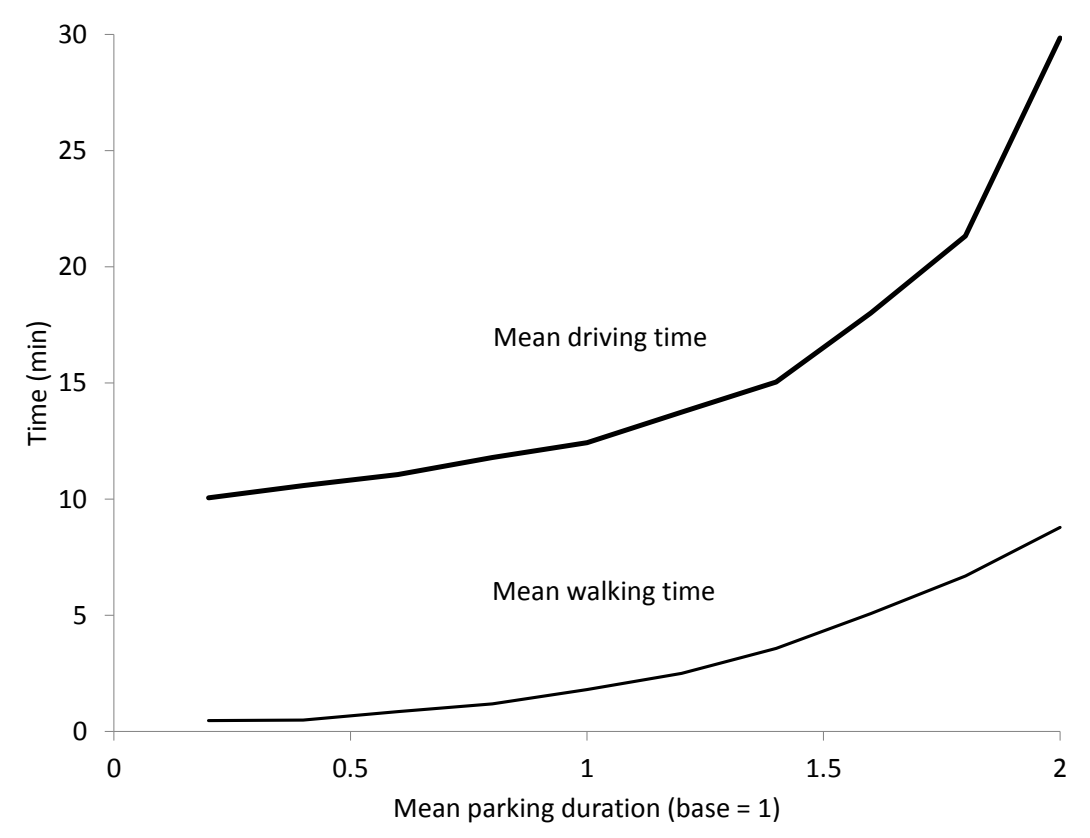

Figure 8: Sensitivity analysis of travel times to mean parking duration

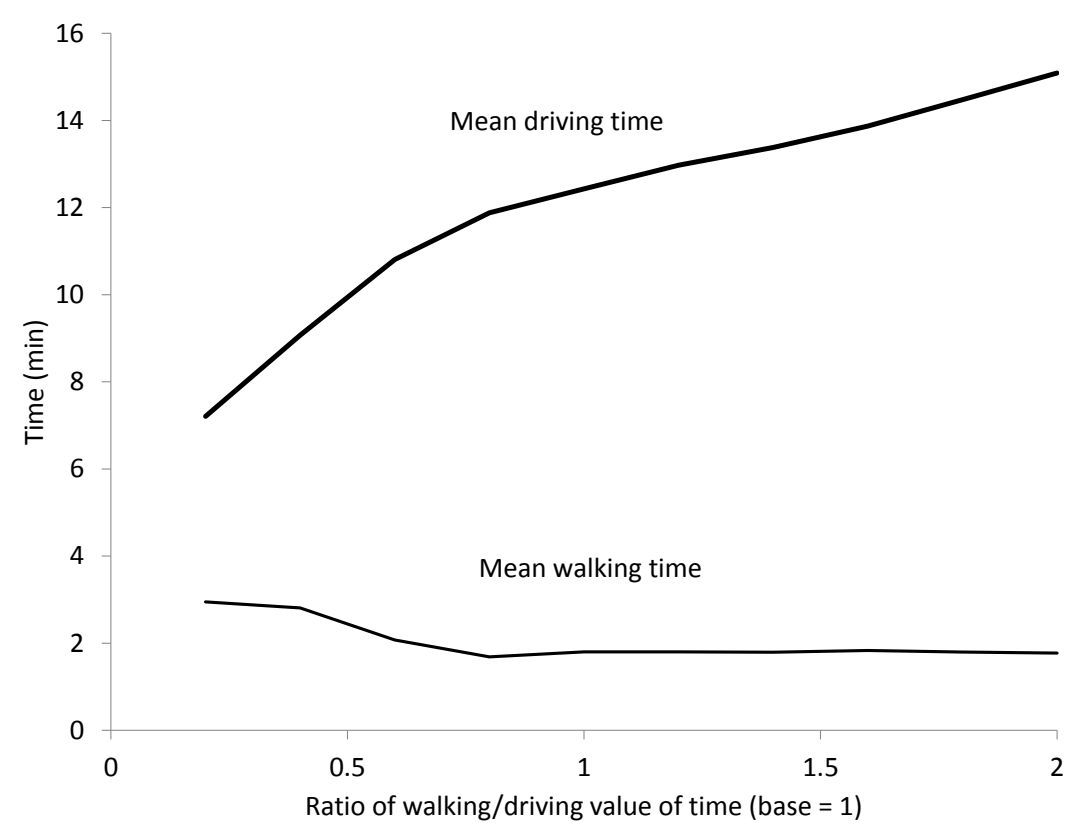

Figure 9: Sensitivity analysis of travel times to value of walking time 
congestion, or destination choice or demand elasticity depending on parking availability. Likewise, the assumption that trips only involve a single stop requiring parking search prevents modeling of trip chains, or parking congestion at the home location. Relaxing these and other assumptions would make valuable topics for future research.

\section{Acknowledgements}

This material is based upon work supported by the National Science Foundation under Grant Nos. 1069141/1157294 and 1254921. Partial support was also provided by the Data-Supported Transportation Planning and Operations University Transportation Center. The authors are grateful for this support. Two anonymous referees provided valuable suggestions on the manuscript which have improved its clarity and precision.

\section{References}

Arnott, R., A. de Palma, and R. Lindsey (1991). Does providing information to drivers reduce traffic congestion? Transportation Research Part A 25(5), 309-318.

Arnott, R. and J. Rowse (1999). Modeling parking. Journal of Urban Economics 45, 97-124.

Axhausen, K. W., J. W. Polak, M. Boltze, and J. Puzicha (1994). Effectiveness of the parking guidance system in Frankfurt am Main. Traffic Engineering and Control 35(5), 304-309.

Bar-Gera, H. (2002). Origin-based algorithm for the traffic assignment problem. Transportation Science 36(4), 398-417.

Bar-Gera, H. (2014). Transportation test problems. Website: http://www.bgu.ac.il/ bargera/ tntp/. Accessed April 28, 2009.

Benenson, I., K. Martens, and S. Birfir (2008). PARKAGENT: an agent-based model of parking in the city. Computers, Environment, and Urban Systems 32, 431-439.

Boyce, D., B. Ralevic-Dekic, and H. Bar-Gera (2004). Convergence of traffic assignments: how much is enough? Journal of Transportation Engineering 130(1), 49-55.

Dieussart, K., K. Aerts, T. Steenberghen, S. Maerivoet, and K. Spitaels (2009). SUSTPARK: an agent-based model for simulating parking search. Presented at the 12th AGILE International Conference on Geographic Information Science, Hannover, Germany.

Eldin, N., T. Y. El-Reedy, and H. K. Ismail (1981). A combined parking and traffic assignment model. Traffic Engineering and Control 10, 524-530.

Facchinei, F. and J. S. Pang (2003). Finite-dimensional variational inequalities and complementarity problems. Springer Verlag.

Gallo, M., L. d'Acierno, and B. Montella (2011). A multilayer model to simulate cruising for parking in urban areas. Transport Policy 18(5), 735-744. 
Gardner, L., S. D. Boyles, and S. T. Waller (2011). Quantifying the benefit of responsive pricing and travel information in the stochastic congestion pricing problem. Transportation Research Part $A$ 45, 204-218.

Gentile, G. (2009). Linear User Cost Equilibrium: a new algorithm for traffic assignment. Working paper.

Glasnapp, J., H. Du, C. Dance, S. Clinchant, A. Pudlin, D. Mitchell, and O. Zoetner (2014). Understanding dynamic pricing for parking lots in Los Angeles: survey and ethnographic results. HCI in Business Lecture Notes in Computer Science 8527, 316-327.

Greenberg, A. (2012). Contemporary approaches to parking pricing: a primer. Technical Report FHWA-HOP-12-026, FHWA Office of Operations, U. S. Department of Transportation.

Hunt, J. D. and S. Teply (1993). A nested logit model of parking location choice. Transportation Research Part B 27(4), 253-265.

Lam, W. H. K., Z. C. Li, H. J. Huang, and S. C. Wong (2006). Modeling time-dependent travel choice problems in road networks with multiple user classes and multiple parking facilities. Transportation Research Part B 40, 368-395.

Lam, W. H. K., M. L. Tam, and M. G. H. Bell (2002). Optimal road tolls and parking charges for balancing the demand and supply of road transport facilities. In M. A. P. Taylor (Ed.), Proceedings of the 15th International Symposium on Transportation and Traffic Theory, pp. 561-582.

Leurent, F. and H. Boujnah (2012). Traffic equilibrium in a network model of parking and route choice, with search circuits and cruising flows. In Proceedings of EWGT2012, Paris. EURO Working Group on Transportation.

Li, Z. C., H. J. Huang, W. H. K. Lam, and S. C. Wong (2007). A model for evaluation of transport policies in multimodal networks with road and parking capacity constraints. Journal of Mathematical Modeling and Algorithms 6, 236-257.

Meng, Q., H. Yang, and M. G. H. Bell (2001). An equivalent continuously differentiable model and a locally convergent algorithm for the continuous network design problem. Transportation Research Part B 35(1), 83-105.

Nie, Y. M. (2011). Multi-class percentile user equilibrium with flow-dependent stochasticity. Transportation Research Part B 45(10), 1641-1659.

Patriksson, M. (1994). The Traffic Assignment Problem - Models and Methods. Utrecht, Netherlands: VSP.

Pierce, G. and D. Shoup (2013). Getting the prices right: an evaluation of pricing parking by demand in San Francisco. Journal of the American Planning Association 79(1), 67-81.

Polycarpou, E., L. Lambrinos, and E. Protopapadakis (2013). Smart parking solutions for urban areas. Presented at the IEEE 14th International Symposium and Workshops on a World of Wireless, Mobile and Multimedia Networks (WoWMoM), Madrid.

Provan, J. S. (2003). A polynomial-time algorithm to find shortest paths with recourse. Networks 41(2), 115-125. 
Qian, Z. S. and R. Rajagopal (2014). Optimal occupancy-driven parking pricing under demand uncertainties and traveler heterogeneity: a stochastic control approach. Transportation Research Part B 67, 144-165.

Qian, Z. S., F. E. Xiao, and H. M. Zhang (2012). Managing morning commute traffic with parking. Transportation Research Part B 46(7), 894-916.

Ross, C. (2013). Boston limiting new parking as number of residences soars. The Boston Globe, July 5 .

Rye, T. (2006). Parking management and competence. Online: http://transportlearning.net/ competence/docs/parking_en.pdf, accessed August 12, 2014.

Shoup, D. C. (2006). Cruising for parking. Transport Policy 13, 479-486.

Tang, S., T. Rambha, R. Hatridge, S. D. Boyles, and A. Unnikrishnan (2014). Modeling parking search on a network using stochastic shortest paths with history dependence. Accepted for publication in Transportation Research Record.

Thompson, R. G. and A. J. Richardson (1998). A parking search model. Transportation Research Part A 32(3), 159-170.

Unnikrishnan, A. and S. T. Waller (2009). User equilibrium with recourse. Networks and Spatial Economics 9(4), 575-593.

Waller, S. T. and A. K. Ziliaskopoulos (2002). On the online shortest path problem with limited arc cost dependencies. Networks 40(4), 216-227.

Yang, H., W. Liu, X. Wang, and X. Zhang (2013). On the morning commute problem with bottleneck congestion and parking space constraints. Transportation Research Part B 58, 106118.

Zhang, X., H.-J. Huang, and H. M. Zhang (2008). Integrated daily commuting patterns and optimal road tolls and parking fees in a linear city. Transportation Research Part B 42(1), 38-56.

Zhang, X., H. Yang, and H.-J. Huang (2011). Improving travel efficiency by parking permits distribution and trading. Transportation Research Part B 45(7), 1018-1034.

\section{Appendix}

In this section we develop one potential specification for the $p_{i j}$ functions. For each link, assume that the total number of parking spaces is given by the positive integer $S_{i j}$. (If no parking is available, $p_{i j}$ is uniformly zero.) Further assume that the mean parking time for any vehicle on this link is $\mu_{i j}$, that the parking duration for any vehicle is exponentially distributed with this mean independent of any other, and that the headways between vehicles arriving to park are also exponentially distributed with mean headway $1 / x_{i j, S}$. Omitting subscripts for brevity, we now derive $p_{i j}$ as follows. 
Define the state variable $P \in\{0, \cdots, S\}$ denoting the number of occupied parking spaces. The evolution of $P$ can be modeled as a Markov chain with transition matrix

$$
\left[\begin{array}{cccccc}
1-x & x & 0 & \cdots & 0 & 0 \\
1 / \mu & 1-x-1 / \mu & x & \cdots & 0 & 0 \\
0 & 2 / \mu & 1-x-1 / \mu & \cdots & 0 & 0 \\
0 & 0 & 3 / \mu & \cdots & 0 & 0 \\
\vdots & \vdots & \vdots & & \vdots & \vdots \\
0 & 0 & 0 & \cdots & 1-(S-1) / \mu-x & x \\
0 & 0 & 0 & \cdots & S / \mu & 1-S / \mu
\end{array}\right]
$$

whose eigenvector gives the steady-state probabilities $\pi_{k}$ for the number of occupied parking spaces. Exploiting the matrix structure, we can calculate this eigenvector recursively:

$$
\begin{aligned}
\frac{\pi_{1}}{\pi_{0}} & =\mu x \\
\frac{\pi_{2}}{\pi_{1}} & =\frac{\mu x}{2} \\
\vdots & \\
\frac{\pi_{n}}{\pi_{n-1}} & =\frac{\mu x}{n} \\
\vdots & \\
\frac{\pi_{S}}{\pi_{S-1}} & =\frac{\mu x}{S}
\end{aligned}
$$

so $\pi_{k} \propto(\mu x)^{k} / k !$ and

$$
\pi_{k}=\frac{(\mu x)^{k} / k !}{\sum_{k^{\prime}=0}^{S}(\mu x)^{k^{\prime}} /\left(k^{\prime}\right) !}
$$

Thus we have

$$
p(x)=1-\pi_{S}=\frac{\sum_{k^{\prime}=0}^{S-1}(\mu x)^{k^{\prime}} /\left(k^{\prime}\right) !}{\sum_{k^{\prime}=0}^{S}(\mu x)^{k^{\prime}} /\left(k^{\prime}\right) !}
$$

With this specification of $p(x), x p(x)$ is strictly increasing as shown below. Application of l'Hospital's rule shows that $C=\lim _{x \rightarrow \infty} x p(x)=S / \mu$ in accordance with intuition. (The maximum rate new vehicles can park is the number of available spaces multiplied by the average rate parked vehicles depart.)

Proposition 14. If parking probabilities are given by (33) then $x p(x)$ is strictly increasing for $x>0$.

Proof. Let $f(x)=x p(x)$. Both the numerator and denominator of $f$ are strictly positive whenever $x$ is; therefore to show that $f^{\prime}(x)>0$ it is enough to show that the numerator of its derivative is strictly positive, that is,

$$
\left(\sum_{k=0}^{S} \frac{\mu^{k}}{k !} x^{k}\right)\left(\sum_{k=0}^{S-1} \frac{\mu^{k}}{(k+1) !} x^{k}\right)-\left(\sum_{k=0}^{S-1} \frac{\mu^{k}}{k !} x^{k}\right)\left(\sum_{k=0}^{S} \frac{\mu^{k}}{(k+1) !} x^{k}\right)>0
$$


Noting that the first term can be replaced by

$$
\left(\sum_{k=0}^{S} \frac{\mu^{k}}{k !} x^{k}\right)\left(\sum_{k=0}^{S} \frac{\mu^{k}}{(k+1) !} x^{k}\right)-\frac{\mu^{S} x^{S}}{(S+1) !}\left(\sum_{k=0}^{S} \frac{\mu^{k}}{(k) !} x^{k}\right)
$$

and the second by

$$
\left(\sum_{k=0}^{S} \frac{\mu^{k}}{k !} x^{k}\right)\left(\sum_{k=0}^{S} \frac{\mu^{k}}{(k+1) !} x^{k}\right)-\frac{\mu^{S} x^{S}}{S !}\left(\sum_{k=0}^{S} \frac{\mu^{k}}{(k+1) !} x^{k}\right)
$$

it is enough to show that

$$
\frac{\mu^{S} x^{S}}{S !}\left(\sum_{k=0}^{S} \frac{\mu^{k}}{(k+1) !} x^{k}\right)>\frac{\mu^{S} x^{S}}{(S+1) !}\left(\sum_{k=0}^{S} \frac{\mu^{k}}{k !} x^{k}\right)
$$

Comparing term by term,

$$
\frac{\mu^{S} x^{S}}{S !} \frac{\mu^{k}}{(k+1) !} x^{k}=\frac{\mu^{S} x^{S}}{(S+1) !} \frac{S+1}{k+1} \frac{\mu^{k}}{k !} x^{k}
$$

Since $S \geq k$ for all terms in the sum (and $S>k$ for all but the last) this establishes the result.

When $S$ is large, the formula (33) may be difficult to evaluate numerically, so an approximation is given here. Define $B_{i}=(\mu x)^{i} / i$ ! so (33) is simply

$$
p(x)=1-\frac{B_{S}}{\sum_{k=0}^{S} B_{k}}
$$

Now $B_{S}=e^{\log B_{S}}$ and

$$
\log B_{k} \approx k \log (\mu x)-k \log k+k-\frac{1}{2} \log (2 \pi k)
$$

using Stirling's approximation to $k$ !. Furthermore, using the Taylor expansion of $e^{x}$ we have

$$
B_{k} \approx 1+k \log (\mu x)-k \log k+k-\frac{1}{2} \log (2 \pi k)
$$

which can be directly substituted in the numerator of (39). In the denominator, using the asymptotic expressions

$$
\sum_{k=1}^{S} \log k \approx\left(S+\frac{1}{2}\right) \log S-S+\frac{1}{2} \log (2 \pi)
$$

and

$$
\sum_{k=1}^{S} k \log k \approx K-\frac{S^{2}}{4}+\frac{S(S+1)}{2} \log S+\frac{\log S}{12}
$$

with $K=\frac{1}{12}-\zeta^{\prime}(-1)$ where $\zeta$ is the Riemann zeta function, we finally obtain

$$
p(x) \approx 1-\frac{1+S\left(\log \frac{\mu x}{S}+1\right)-\frac{1}{2} \log (2 \pi S)}{1-K+S-\frac{1}{4} \log (8 \pi S)+\frac{S(S+1)}{2}\left(\log \frac{\mu x}{S}+1\right)+\frac{S^{2}}{4}+\frac{S}{2}(1-\log (2 \pi S))}
$$

after some algebra. While an approximation, this function is easier to evaluate for large $S$ (say, if a parking link represents a large lot.) 